\title{
Impact of micro-scale residual stress on in-situ tensile testing of ductile cast iron: Digital volume correlation vs. model with fully resolved microstructure vs. periodic unit cell
}

Andriollo, Tito; Zhang, Yubin; Fæster, Søren; Thorborg, Jesper; Hattel, Jesper

Published in:

Journal of the Mechanics and Physics of Solids

Link to article, DOI:

10.1016/j.jmps.2019.01.021

Publication date:

2019

Document Version

Peer reviewed version

Link back to DTU Orbit

Citation (APA):

Andriollo, T., Zhang, Y., Fæster, S., Thorborg, J., \& Hattel, J. (2019). Impact of micro-scale residual stress on insitu tensile testing of ductile cast iron: Digital volume correlation vs. model with fully resolved microstructure vs. periodic unit cell. Journal of the Mechanics and Physics of Solids, 125, 714-735.

https://doi.org/10.1016/j.jmps.2019.01.021

\section{General rights}

Copyright and moral rights for the publications made accessible in the public portal are retained by the authors and/or other copyright owners and it is a condition of accessing publications that users recognise and abide by the legal requirements associated with these rights.

- Users may download and print one copy of any publication from the public portal for the purpose of private study or research.

- You may not further distribute the material or use it for any profit-making activity or commercial gain

- You may freely distribute the URL identifying the publication in the public portal 


\section{Impact of micro-scale residual stress on in-situ tensile testing of ductile cast iron: Digital volume correlation vs. model with fully resolved microstructure vs. periodic unit cell}

Tito Andriollo ${ }^{\mathrm{a}, \mathrm{d}}$, Yubin Zhang ${ }^{\mathrm{a}}$, Søren Fæster ${ }^{\mathrm{b}}$, Jesper Thorborg ${ }^{\mathrm{a}, \mathrm{c}}$ and Jesper Hattel

a Department of Mechanical Engineering, Technical University of Denmark, DK-2800 Kgs. Lyngby, Denmark

b Department of Wind Energy, Technical University of Denmark, Risø Campus Frederiksborgvej 399, DK-4000 Roskilde, Denmark

c MAGMA GmbH, D-52072 Aachen, Germany

d Corresponding author. Address: Technical University of Denmark, Produktionstorvet, Building 425, room 225, 2800 Kgs. Lyngby, Denmark. Phone: +45 452547 22. E-mail: titoan@mek.dtu.dk

\section{Abstract}

The understanding of the mechanisms controlling deformation of ductile iron at the microscale and their coupling to the manufacturing conditions is still far from complete. In this respect, recent synchrotron-based studies have demonstrated that the thermal contraction mismatch between the graphite particles and the matrix during solid-state cooling leads to a complex residual stress state in the microstructure. To investigate its impact on the roomtemperature tensile deformation, a computational-experimental analysis extendable to other similar composite materials is presented in this paper. First, a miniaturized specimen is loaded and imaged in-situ with X-ray tomography. Then, the microscale displacement is reconstructed using digital volume correlation (DVC) and used to prescribe the boundary conditions in a finite element model of the full microstructure between two cross-sections. The model predictions at both the macroscale - tensile force and lateral contraction - and the microscale - strain field - are compared to the corresponding experimental and DVCbased data for several choices of the initial stress state, particles' mechanical behavior and strength of the particles-matrix interface. It is proved that the micro-scale residual stress 
and a low interface strength are the key to explain the early stages of the tensile deformation of ductile iron. Finally, it is shown that a simple unit cell model of the microstructure would lead to significantly different results, thus demonstrating the superior accuracy and robustness of the present approach.

\section{Graphical abstract}
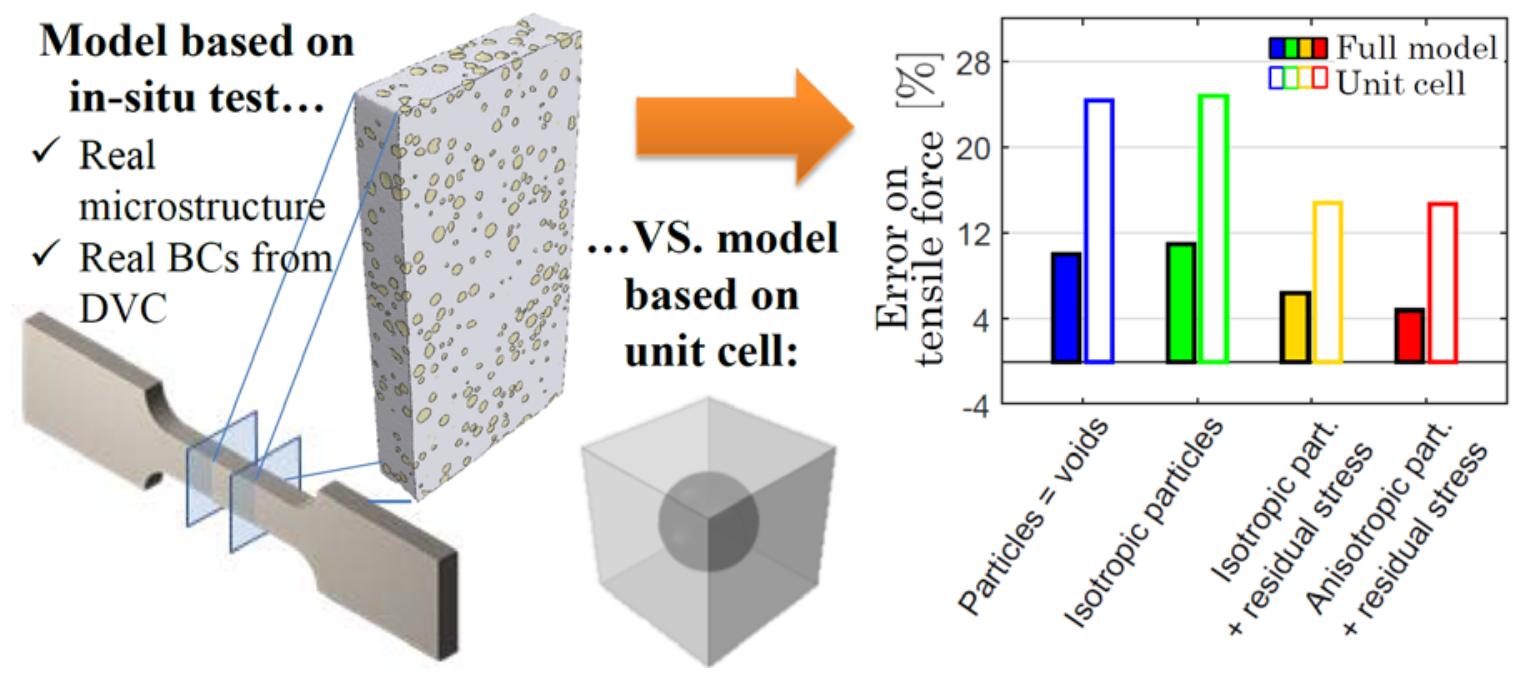

\section{Keywords}

In-situ test; Digital volume correlation; Cast iron; Composite; Residual stress;

\section{Contents}

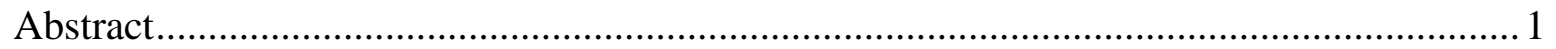

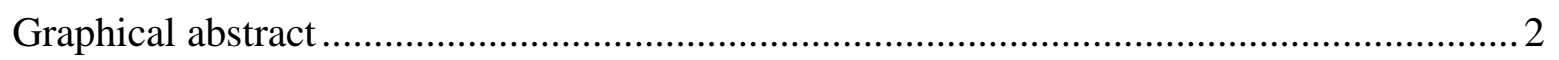

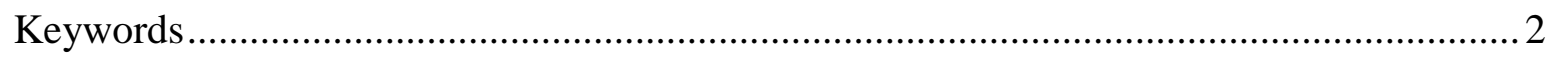

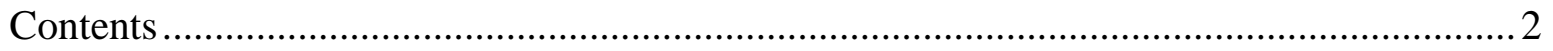

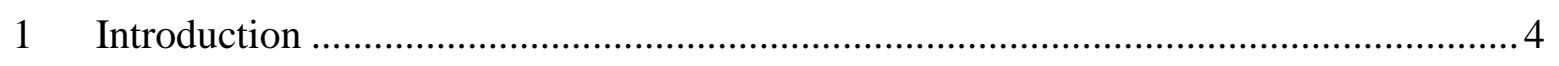

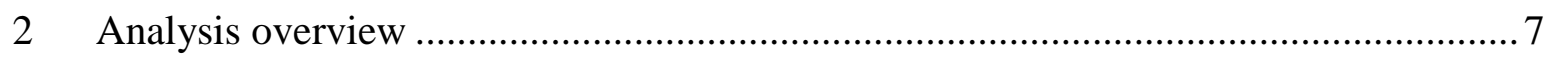

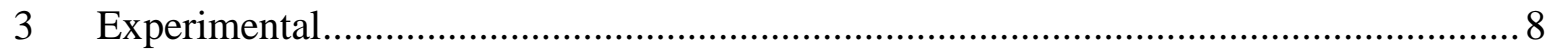

3.1 In-situ tensile testing with X-ray tomography ............................................ 8 
3.2 Digital volume correlation ......................................................................... 10

3.2.1 Choice of the subvolume size ....................................................................... 10

3.2.2 Analysis of the microscopic displacement field ............................................ 13

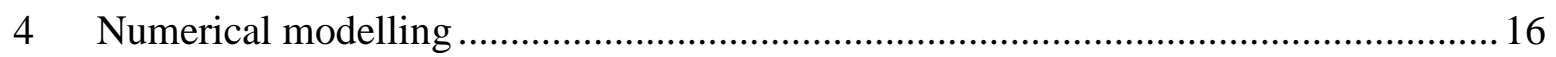

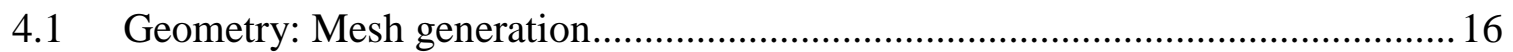

4.2 Mechanical behavior of the matrix-particles interface …………………………... 17

4.3 Mechanical behavior of the matrix …………………............................................. 19

4.4 Mechanical behavior of the particles ....................................................................... 21

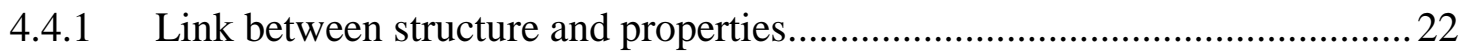

4.4.2 Implementation of an anisotropic thermo-elastic model .................................. 23

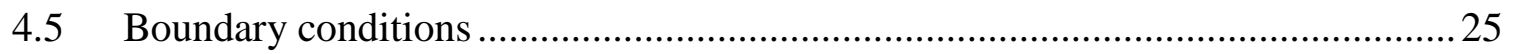

4.5.1 Formation of micro-scale residual stresses.................................................... 25

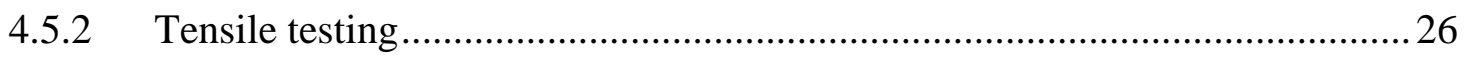

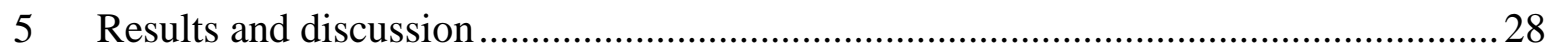

5.1 Full model vs. experiments: Macroscale comparison..............................................28

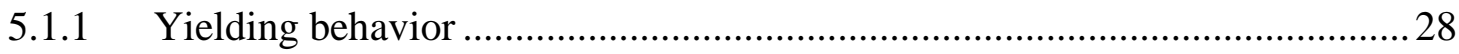

5.1.2 Hardening behavior .................................................................................... 32

5.2 Full model vs. experiments: Microscale comparison ................................................ 35

$5.3 \quad$ Full model vs. unit cell model .............................................................................. 38

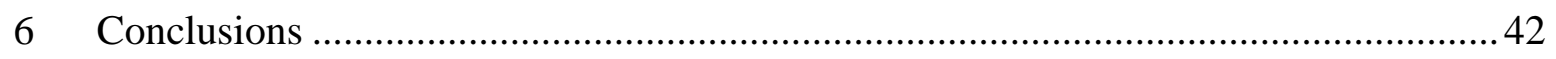

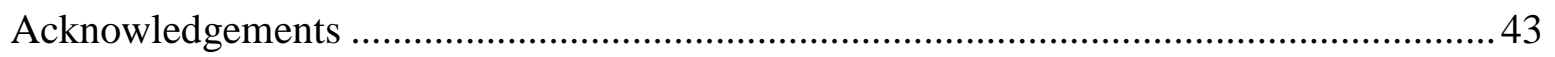

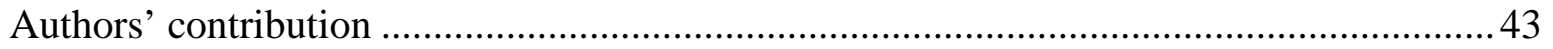

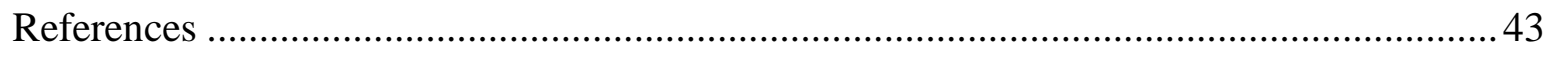




\section{Introduction}

Modern ductile cast iron (DCI) is an advanced structural material heavily used in key industrial sectors like automotive and energy production, which, alone, accounts for as much as $25 \%$ of the castings produced worldwide ("50th Census of World Casting Production,” 2016). Due to its high technological importance, a number of studies have focused on the mechanisms controlling deformation and fracture at the microscale in the past decades. Nevertheless, as emphasized in a recent review paper (Hütter et al., 2015), a complete understanding of the behavior of DCI during both monotonic and cyclic loading has not been achieved yet. One of the main reasons is the challenge represented by the very complex material microstructure, which consists of graphite particles embedded in a steel matrix where multiple phases may coexist depending on chemistry and processing conditions. In this respect, recent synchrotron-based studies by the present authors have revealed, for the first time, that the thermal contraction mismatch between the particles and the matrix during solid-state cooling leads to localized residual elastic and plastic deformation in the matrix (Zhang et al., 2019, 2016). Remarkably, strong fluctuations of the residual elastic strain exist in proximity to the particles, which have been explained in terms of their highly inhomogeneous, anisotropic internal structure. Subsequent numerical simulations have suggested that the peak stress corresponding to this complex residual deformation can be as high as the material macroscopic yield strength (Andriollo et al., 2018b) and the plastic flow of the matrix around the particles can affect the coefficient of thermal expansion at the macro-scale (Rodriguez et al., 2018). In light of these findings, a natural question then arises as to how and how much this micro-scale residual stress field forming during solid-state cooling can affect the mechanical response of DCI during room temperature loading.

Traditionally, the impact of similar micro-mechanical phenomena in DCI has been studied with techniques developed for metal matrix composites (MMCs), due to the strong similarities between these two material classes (Grimvall, 1997). Concerning this, early attempts to estimate the mechanical properties as a function of the properties and geometry of the individual MMCs' constituents relied on standard analytical methods for mean-field 
homogenization. Such methods are often based on the well-known Eshelby's elastic solution to the problem of an ellipsoidal inclusion embedded into an infinite medium (Eshelby, 1957). A recent account on the application of mean-field approaches to MMCs has emphasized that they represent, due to their simplicity, a fast and efficient tool for preliminary design of novel MMCs (Kursa et al., 2018). Nevertheless, when a full resolution of the local fields is needed to investigate localization, damage and failure mechanisms at the microstructural level, analytical mean-field approaches become inadequate. Numerical techniques which allow for the latter type of analyses are normally based on the concept of representative volume element (RVE), which is intuitively defined as a material volume whose effective behavior is representative of that of the material as a whole (Aboudi et al., 2012; Hill, 1963). Using the RVE as starting point, several numerical homogenization procedures have been proposed to correlate micro- to macro-scale properties, e.g. (Feyel and Chaboche, 2000; Kouznetsova et al., 2001; Matouš et al., 2017; Toro et al., 2016). The main limitation of methods based on the RVE lies in the definition of the appropriate size of the RVE, which has to be sufficiently large to guarantee statistical representativeness, yet small compared to the characteristic dimension of the macroscopic component. Normally, the size of the RVE is determined via an a posteriori analysis referred to as successive sample enlargement (Bargmann et al., 2018). However, this process, besides being time consuming, can fail to provide an outcome when the RVE concept does not hold, which can be the case if either the condition of separation of scale is not fulfilled, or the material microstructure is not statistically homogeneous (OstojaStarzewski, 2006). This last aspect is particularly critical for DCI, as unexpected spatial variations in the graphite size, shape and distribution often occur as a result of local differences in the solidification conditions, associated with e.g. strong local chemical gradients and/or presence of impurities (Azeem et al., 2018; Muhmond and Fredriksson, 2014).

The non-trivial definition of a RVE in DCI - and, more in general, in all cast iron types raises the problem of how to study the micro-mechanical behavior in the non-linear loading regime, during which local microstructural variations - e.g. defects like graphite particles deviating markedly from the expected shape - can play a major role. As a matter of fact, 
unit cell models which circumvent this issue by considering an idealized representation of the microstructure are a common choice (Andriollo et al., 2015; Bonora et al., 2005; Collini and Nicoletto, 2005; Iannitti et al., 2017; Pina et al., 2015; Rabold and Kuna, 2005). Apart from these, procedures based on statistical approaches that avoid defining a RVE and work with smaller volume elements have been proposed (Metzger and Seifert, 2015). Attempts to define 2D RVEs have been made also (Fernandino et al., 2017; Pina et al., 2016; Zhang et al., 2018), even though the reduced model dimensionality might be limiting for materials whose microstructure is inherently three dimensional. A promising approach, initially proposed in a context more general than DCI, is to develop combined experimentalcomputational procedures taking advantage of the recent progress in X-ray computed tomography and digital volume correlation (DVC) (Hild et al., 2016; Mostafavi et al., 2016). The underlying idea is to perform a series of tomographic reconstructions of a given material volume during a mechanical test. By applying DVC algorithms, the mechanical displacement field can be reconstructed and used as boundary conditions in a numerical model. Furthermore, if the volume under consideration is sufficiently large to include the region where the external load is applied, the outcome of the model can be compared to a direct force measurement. Recently, (Buljac et al., 2018) applied this innovative approach to DCI, even though the authors neglected completely the composite nature of the material - together with all the effects associated with the interaction between the microstructural constituents, including the formation of residual stresses - by assimilating the graphite particles to voids. Still, it is the present authors' opinion that this type of approach remains potentially very valuable, as it bypasses the need of defining a RVE without compromising the geometrical representation of the microstructure.

Taking the approach just mentioned as source of inspiration, the present paper presents an experimental-computational analysis whose aim is threefold. First, to understand the impact of the manufacturing-induced micro-scale residual stress revealed in (Zhang et al., 2019, 2016) on the tensile deformation of DCI. Second, to assess the sensitivity of the results to apparently critical but scarcely investigated factors like the mechanical behavior of the graphite particles and the strength of the particle-matrix interface. Third, to check whether 
this approach allows a gain in accuracy compared to a much simpler periodic unit cell model that justifies the greater effort in terms of time and resources.

The paper is organized as follows. In section 2, an overview of all the steps involved in the analysis is given. Section 3 describes the in-situ tensile test performed on a miniaturized DCI specimen and the DVC procedure employed to reconstruct the displacement field. Section 4 contains the detail of the finite element model used to simulate manufacturing and tensile loading of the specimen. Finally, section 5 presents the comparison between model predictions and experimental data at both the macro and micro-scale, as well as the comparison with the results obtained from a simple unit cell model.

\section{Analysis overview}

An overview of all the steps involved in the present experimental-computational analysis is given in figure 1 . As the figure shows, the analysis starts with the extraction of a miniaturized tensile specimen from a large DCI component used in a wind turbine. The specimen is placed on a tensile stage and strained incrementally. At each increment, the specimen is imaged in-situ via X-ray computed tomography (CT), in order to monitor the evolution of its microstructure, and DVC is employed to reconstruct the microscopic displacement field associated with the mechanical deformation. Subsequently, a 3D finite element model of the volume of the specimen between two cross-sections is created based on the real microstructure. In a first step, the model is used to simulate the formation of residual stresses during the manufacturing process. After that, the straining history the specimen was subjected to is simulated by applying the microscopic displacement field determined via DVC as boundary conditions. The model predictions at both the macroscale - tensile force and lateral contraction - and the microscale - strain field - are then compared to the corresponding experimental and DVC-based data for several choices of the initial stress state, particles' mechanical behavior and strength of the particles-matrix interface. Finally, a comparison is also made with the predictions of a simplified periodic unit cell model.

In this context, the so-called preparatory steps enclosed by a box in figure 1 aim at providing the models - both the one with the fully resolved microstructure, henceforth 
denoted as the "full" model, as well as the one based on the periodic unit cell - with information concerning the properties of the DCI constituents and the cooling rate experienced by the specimen during manufacturing. Due to the complexity of the procedures employed, the details of the preparatory steps are reported in separate publications and are not repeated here. In particular, the mechanical behavior of the graphite particles was investigated via nano-indentation in (Andriollo et al., 2018a) and the constitutive response of the matrix at various temperatures was determined by manufacturing and testing an alloy possessing equivalent metallurgical features in (Andriollo et al., 2018b). Simulation of the manufacturing process of the full-size DCI component, considering DCI as a homogeneous material, was addressed in (Sonne et al., 2017).

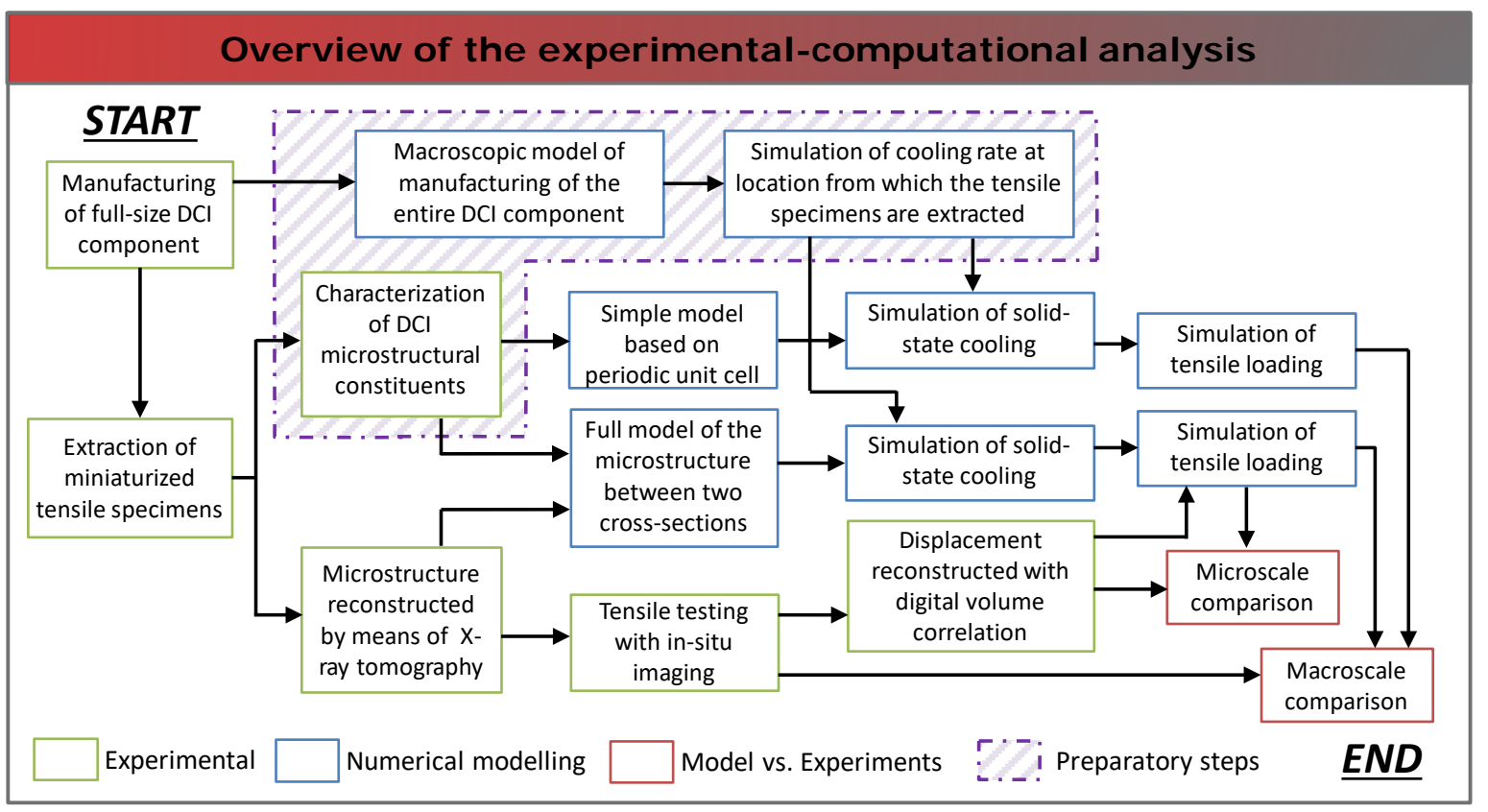

Figure 1 - Overview of the steps involved in the present experimental-computational analysis.

\section{Experimental}

\subsection{In-situ tensile testing with $X$-ray tomography}

A dog bone shaped specimen with gauge length $10 \mathrm{~mm}$ and $1 \mathrm{x} 2 \mathrm{~mm}$ rectangular crosssection was extracted from a large wind turbine component made of DCI, see figure 2 (a). 
Characterization of a small piece of material taken from the same location revealed near spherical graphite particles with mean size $30 \mu \mathrm{m}$ and volume fraction $\approx 11.5 \%$ embedded in a relatively homogeneous matrix of ferrite - mean grain size $30 \mu \mathrm{m}$ - with $\approx 5 \%$ pearlite (Zhang et al., 2016). The specimen was mounted on a displacement-controlled tensile stage equipped with a load cell inside a Zeiss Xradia 520 Versa CT system. Two separate CT scans of the specimen in the unloaded configuration were initially acquired, in order to evaluate the resolution of the DVC procedure - see section 3.2. To this end, polychromatic and conical X-ray beams with energies up to $150 \mathrm{keV}$ were used, resulting in reconstructed density maps with voxel size of $1.359 \mu \mathrm{m}$. Subsequently, three displacement increments were applied in sequence and CT scans were acquired in between, in order to reconstruct the progressive deformation of the specimen microstructure. The displacement magnitude was chosen to produce a stress - defined as the ratio between tensile force and initial area of the cross-section - of about $1 / 2$ of the macroscopic yield stress $\sigma_{y}(0.2 \%$ proof stress) during increment 1 , of about $\sigma_{y}$ during increment 2 and of about $1.5 \sigma_{y}$ during increment 3 . The stress vs. strain response generated in this way is reported in figure 2 (b).

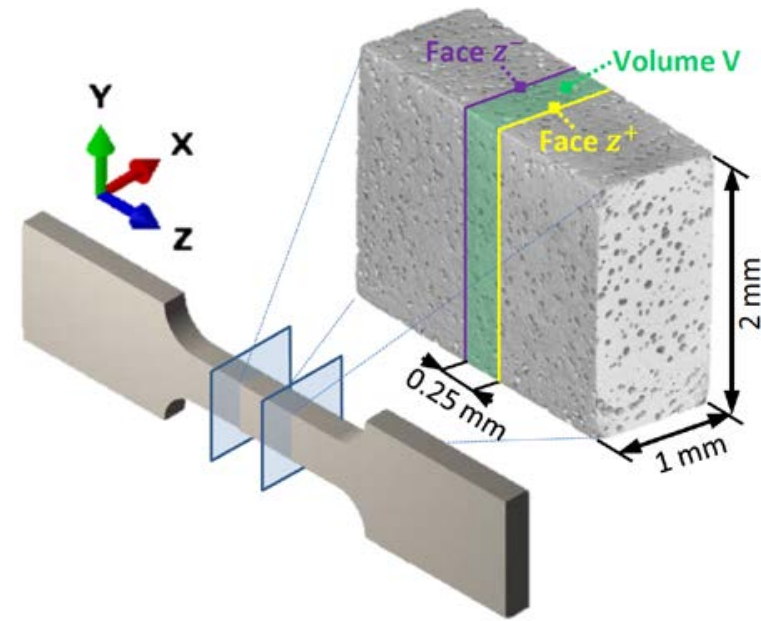

(a)

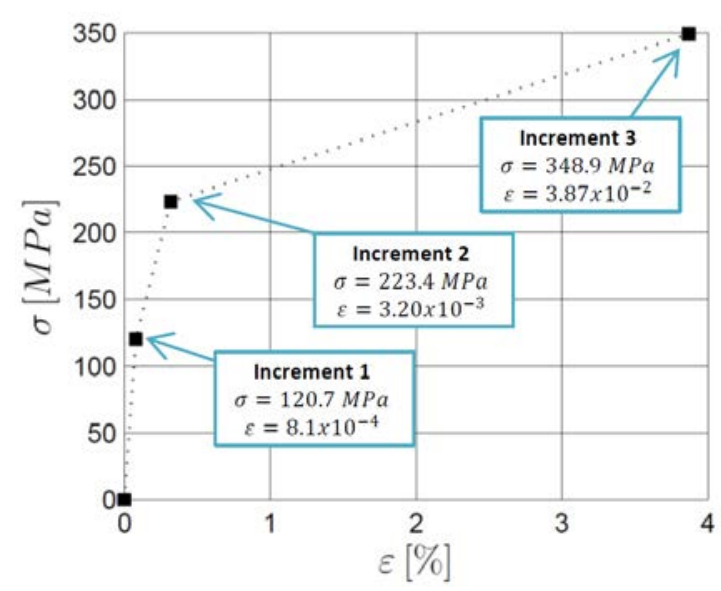

(b)

Figure 2 - In-situ tensile testing of DCI. (a) Geometry of the tensile specimen and example of gray-level density map obtained via CT. The region delimited by the two cross-sections marked as "face $z^{+}$" and "face $z^{-}$, which is highlighted in green, corresponds to the volume V considered in the finite element model. (b) Macroscopic uniaxial stress and strain in the specimen at each increment. 


\subsection{Digital volume correlation}

The subset-based DVC algorithm available in the XVolumeCorrelation extension of the software Avizo ${ }^{\circledR}$ was employed to determine the microscopic displacement field over the volume of the specimen between the two cross-sections $250 \mu \mathrm{m}$ apart marked as face $z^{+}$ and face $z^{-}$in figure 2 (a). This volume - denoted as volume $\mathrm{V}$ - is the region considered in the full finite element model described in section 4.1. As extensively discussed elsewhere, e.g. (Lava et al., 2009), in a subset-based method a matching between two speckle patterns is accomplished by considering a voxel and its neighborhood (usually called subset, or subvolume) in the undeformed image and searching the same subvolume in the deformed image. Accordingly, the region of the reconstructed CT density map corresponding to volume $\mathrm{V}$ was divided into a grid of cubic subvolumes. As the displacement vector is defined only at the center of each subvolume, an additional layer of subvolumes was added along the specimen straining direction - the z-axis in figure 2 (a) and the entire grid was shifted by half the subvolume size to ensure that the centers of the subvolumes forming the first and last layer were lying precisely on the $z^{+}$and $z^{-}$faces.

\subsubsection{Choice of the subvolume size}

At first, an analysis of the influence of the subvolume size was carried out, as it is wellknown that smaller subvolumes provide better spatial resolution, but at the price of a higher uncertainty on the calculated displacement. Accordingly, the DVC algorithm was run considering the two CT maps of the specimen in the unloaded configuration and four different subvolume sizes: $250 \mu \mathrm{m} / 2=125 \mu \mathrm{m}, 250 \mu \mathrm{m} / 3=83.3 \mu \mathrm{m}, 250 \mu \mathrm{m} / 4=62.5 \mu \mathrm{m}$ and $250 \mu \mathrm{m} / 5=50 \mu \mathrm{m}$. As no physical deformation is involved in this case, the standard deviation of the displacement field provides an estimate of the uncertainty associated with the DVC procedure (Morgeneyer et al., 2013). From figure 3, which reports the standard deviation of the displacement norm as a function of the subvolume size, it can be concluded that when the latter is increased from 50 to $125 \mu \mathrm{m}$ the uncertainty decreases by as much as a factor of 7. On the other hand, it should be borne in mind that the characteristic size of the DCI microstructure is approximately $30 \mu \mathrm{m}$, i.e. the mean size of the graphite particles, 
meaning that local fluctuations of the displacement field due to microstructural features can hardly be captured with subvolumes much larger than this value.

To select the subvolume size that offers the best compromise, the impact of the displacement uncertainty on the DVC-based estimate of the macroscopic deformation of the volume $\mathrm{V}$ was analyzed. In this respect, it is noted that the macroscopic strain increment $\Delta \bar{\varepsilon}_{z z}$ experienced by the volume $\mathrm{V}$ can be associated with the volumetric average of the corresponding microscopic quantity $\Delta \varepsilon_{z z}$ :

$$
\Delta \bar{\varepsilon}_{z Z} \approx \frac{1}{\mathrm{~V}} \int_{V} \Delta \varepsilon_{z z} d V
$$

Due to the small entity of the deformation, the infinitesimal definition of strain can be employed. Furthermore, it can be assumed that the lateral surfaces of V, which are part of the free surface of the specimen, are normal to the z-axis pointwise. Then, it is easily shown that

$$
\Delta \bar{\varepsilon}_{z z} \approx \frac{1}{L_{z}}\left(u_{z}^{a v g, z+}-u_{z}^{a v g, z-}\right)
$$

where $L_{z}$ is the length of $\mathrm{V}$ along the $\mathrm{z}$-axis and $u_{z}^{a v g, z+}-$ resp. $u_{z}^{a v g, z-}-$ is the average zdisplacement over the $z^{+}$face - resp. over the $z^{-}$face - during the strain increment considered. As the DVC displacement is known at the center of the subvolumes, it follows that

$$
\Delta \bar{\varepsilon}_{z z} \approx \frac{1}{L_{z}}\left[\frac{1}{n_{x} n_{y}} \sum_{n=1}^{n_{x} \times n_{y}}\left(u_{z, n}^{z+}-u_{z, n}^{z-}\right)\right]
$$

where $n_{x}$ and $n_{y}$ denote the number of subvolumes along the x- and y-axis. Equation (3) suggests that when DVC is applied to the two CT maps of the specimen in the unloaded configuration, the uncertainty $\delta \bar{\varepsilon}_{z Z}$ on the estimate of the macroscopic deformation of $\mathrm{V}$ can be assessed as

$$
\delta \bar{\varepsilon}_{z z} \approx \frac{1}{L_{z}} \operatorname{StDev}\left(u_{z, n}^{z+}-u_{z, n}^{z-}\right)
$$

The dependence on the subvolume size of the term multiplying $L_{z}$ on the right-hand-side of the last equation is plotted in figure 3 , where a trend similar to that of the standard deviation of the displacement norm is observed. Table 1 reports the ratio between the magnitude $\Delta \varepsilon$ 
of the uniaxial strain increments applied during the tensile test and $\delta \bar{\varepsilon}_{z z}$, for different choices of the subvolume size. It can be observed that while this quantity is always much greater than 1 for the third strain increment, due to the significant deformation undergone by the specimen, values close to unity are obtained for the other two increments. In particular, values below one are seen for the first increment relative to all subvolume sizes except $125 \mu \mathrm{m}$, which, on the other hand, is approx. 4 times larger than the characteristic size of the microstructure. According to equation (4), the situation could be improved by increasing $L_{z}$, in order to reduce $\delta \bar{\varepsilon}_{z z}$. However, the selected value of $250 \mu \mathrm{m}$ represents the limit beyond which the finite element model of the volume $\mathrm{V}$ - see section 4 - would become computationally intractable with the available hardware. Therefore, it was decided to merge the first and second increment, in order to increase $\Delta \varepsilon$ and, consequently, the ratio $\Delta \varepsilon / \delta \bar{\varepsilon}_{z z}$. The last row of table 1 shows that, with this operation, the values of $\Delta \varepsilon / \delta \bar{\varepsilon}_{z Z}$ for the two smallest subvolumes remain close to unity, but a more reassuring value of 2.64 is obtained for the subvolume with size $83.3 \mu \mathrm{m}$. This means that, if the increments 1 and 2 are combined, this subvolume size can be considered a compromise: it delivers sufficient accuracy, at the same time allowing a gain of $33 \%$ in spatial resolution compared to the $125 \mu \mathrm{m}$ option. For this reason, it was eventually selected for the present investigation.

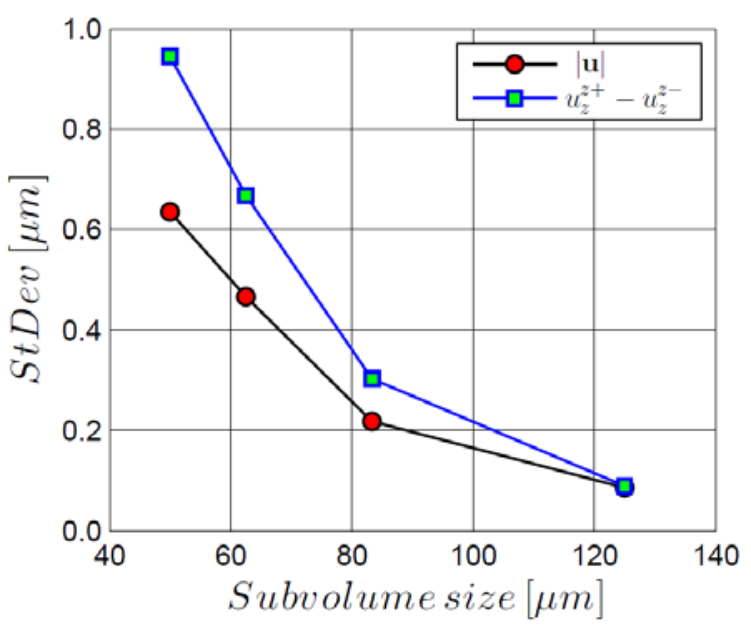

Figure 3 - Effect of the subvolume size on the uncertainty of the DVC procedure, expressed as the standard deviation of the norm of the displacement $|\boldsymbol{u}|$ and of the difference $u_{z}^{z+}-u_{z}^{z-}$ between the z-component of the displacement on the $z^{+}$and $z^{-}$faces. 
Table 1: Ratio between the magnitude $\Delta \varepsilon$ of the uniaxial strain increments imposed to the specimen and the uncertainty $\delta \bar{\varepsilon}_{z z}$ on the DVC-based macroscopic strain of volume V estimated via equation (4).

\begin{tabular}{ccccc}
\hline & $\begin{array}{c}\text { DVC subvolume } \\
\text { size } 125.0 \mu \mathrm{m}\end{array}$ & $\begin{array}{c}\text { DVC subvolume } \\
\text { size } 83.3 \mu \mathrm{m}\end{array}$ & $\begin{array}{c}\text { DVC subvolume } \\
\text { size } 62.5 \mu \mathrm{m}\end{array}$ & $\begin{array}{c}\text { DVC subvolume } \\
\text { size } 50.0 \mu \mathrm{m}\end{array}$ \\
\hline Increment 1 & 2.26 & 0.67 & 0.30 & 0.21 \\
Increment 2 & 6.67 & 1.97 & 0.89 & 0.63 \\
Increment 3 & 98.77 & 29.21 & 13.25 & 9.37 \\
Combined 1\&2 & 8.93 & 2.64 & 1.20 & 0.85 \\
\hline
\end{tabular}

\subsubsection{Analysis of the microscopic displacement field}

The displacement field $\boldsymbol{u}$ over the entire volume $\mathrm{V}$ was fitted with a linear function $\boldsymbol{u}^{\boldsymbol{L}}$ of the type

$$
\boldsymbol{u}^{L}=\boldsymbol{u}^{C}+\mathbf{A} \cdot \boldsymbol{x}
$$

where $\boldsymbol{u}^{\boldsymbol{C}}$ and $\boldsymbol{x}$ are the displacement of and the distance from the center of $\mathrm{V}$, respectively, and $\mathbf{A}$ is a 3x3 matrix. The part of the displacement responsible for inducing strain in the material $\boldsymbol{u}^{\boldsymbol{S}}$ was estimated as

$$
\boldsymbol{u}^{S}=\boldsymbol{u}-\boldsymbol{u}^{\boldsymbol{C}}-\frac{1}{2}\left(\mathrm{~A}-\mathrm{A}^{\mathrm{T}}\right) \cdot \boldsymbol{x}
$$

with $(\cdot)^{\mathbf{T}}$ denoting the transposition operator. Figure 4 (a) and (b) show the z-component of $\boldsymbol{u}^{\boldsymbol{S}}$ over both the $z^{-}$and the $z^{+}$face for the combined increment 1\&2. Some odd values can be noted close to the free surface, which could be either a consequence of localized surfacerelated phenomena occurring upon load application or artifacts due to poor correlation. Remarkably, the difference between the average value of the z-component of $\boldsymbol{u}^{\boldsymbol{S}}$ in figure 4 (a) and that in figure 4 (b) is $0.79 \mu \mathrm{m}$, which, when divided by thickness of volume $\mathrm{V}$, provides a strain estimate of $3.16 \times 10^{-3}$. This value differs from the macroscopic strain applied to the specimen by only $1.3 \%$, see figure 2 (b), thus supporting the conclusion of the previous section that subvolumes with size $83.3 \mu \mathrm{m}$ provide a sufficient level of accuracy.

For the increment 3, the patterns of the z-component of $\boldsymbol{u}^{\boldsymbol{S}}$ over the $z^{-}$and the $z^{+}$face (see figure 4 (c) and (d)) suggest that a certain amount of spurious bending about the $\mathrm{x}$-axis took place. In order to check the extent of this phenomenon, both patterns were fitted with a 
plane and the inclination of the latter with respect to the $x-y$ plane was calculated. Multiplication of the difference in the planes' inclination by half the size of the specimen cross-section along the $y$-axis yielded a value of $0.88 \mu \mathrm{m}$. This is one order of magnitude smaller than the difference between the average z-displacement of the $z^{-}$face and that of the $z^{+}$face, meaning that the contribution of bending to the deformation of volume $\mathrm{V}$ is quite small, even though not negligible. Concerning this, it is worth emphasizing that the presence of bending it not expected to affect the outcome of the present study. Indeed, one of the major advantages of the present approach is that it is insensitive to spurious deformation modes, as DVC allows using the real microscopic displacement experienced by the volume $\mathrm{V}$ as boundary conditions in the full model. 


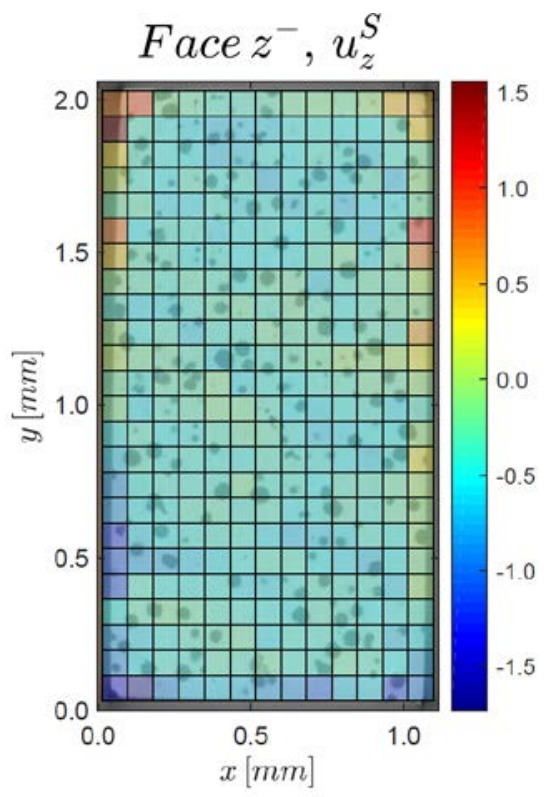

(a)

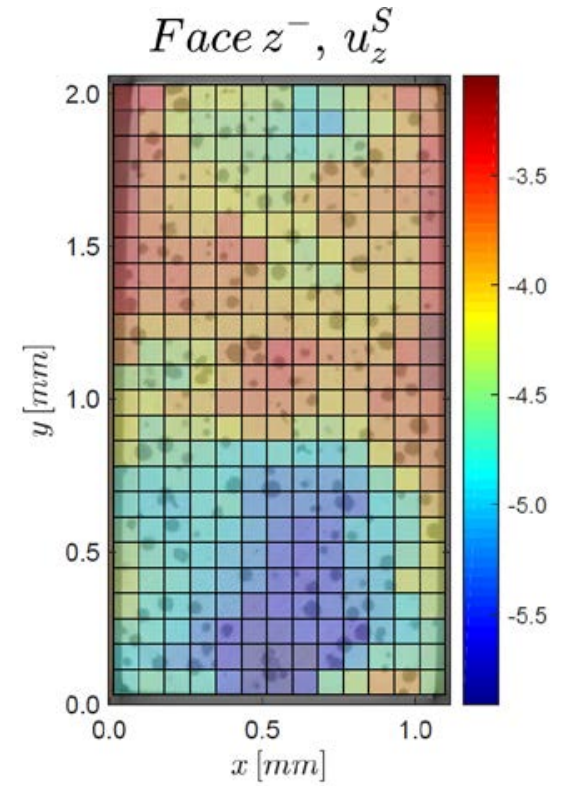

(c)

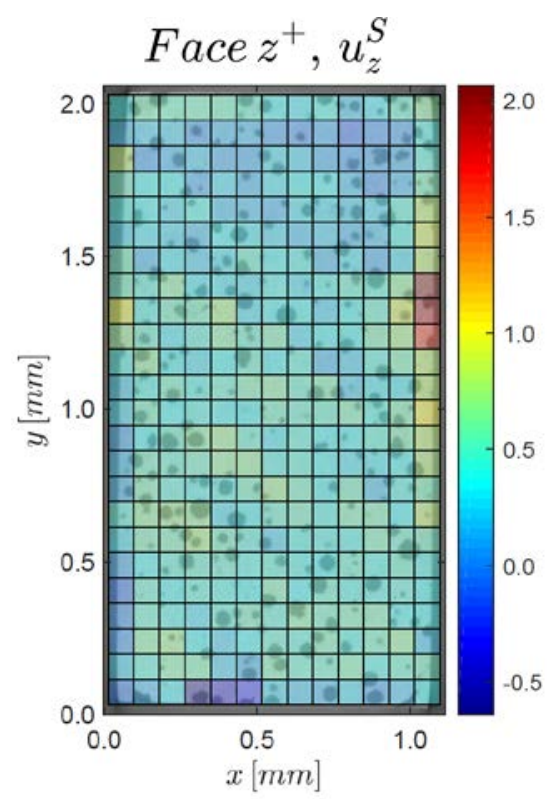

(b)

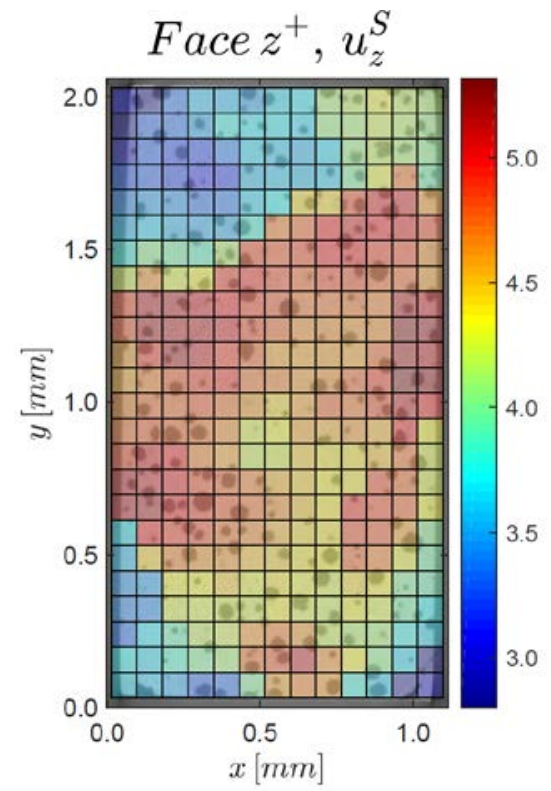

(d)

Figure 4 - Contours of the z-component of the displacement reconstructed via DVC using a subvolume size of $83.3 \mu \mathrm{m}$, superimposed to the real microstructure. (a-b) Combined increment 1\&2. (c-d) Increment 3. Units are $\mu \mathrm{m}$. The rigid part of the displacement has been removed from all the contours, see eq. (6). 


\section{Numerical modelling}

This section presents the details of the full model, with special focus on the techniques used to account for variable strength of the particles-matrix interface and different types of mechanical behavior of the particles. The details of the periodic unit cell model based on a simple cubic cell can be found in (Andriollo et al., 2018c). It is worth remarking that these two models differ in terms of the geometry and the way the boundary conditions are applied, but all the other features like e.g. constitutive material behaviors, particles-matrix interface type, etc. are the same.

\subsection{Geometry: Mesh generation}

The region of the CT density map corresponding to the volume $\mathrm{V}$ of the specimen in the unloaded configuration was used as starting point. In order to generate a mesh conforming to the DCI microstructure, the meshing toolbox of the software Avizo® ${ }^{\circledR}$ was employed. To begin with, a median filter was applied to remove the noise, followed by segmentation based on thresholding to identify the DCI matrix. Subsequently, the graphite was separated from the air surrounding the specimen via a closing operation, as other segmentation techniques proved ineffective due to the poor contrast between these two phases. During this step, graphite particles with equivalent diameter less than $10 \mu \mathrm{m}$ were discarded and considered as part of the matrix, as these are likely either inclusions or porosities (Lacaze et al., 1998; Mukherjee et al., 2017; Pedersen and Tiedje, 2008). It is worth remarking that this choice is not expected to affect the behavior of the material significantly, at least within the small deformation range investigated here, as the volume fraction of the former particles is very low. Finally, on the basis of the segmented data, which is visualized in

figure 5 , two $3 \mathrm{D}$ conforming meshes with $\approx 2 \times 10^{6}$ and $\approx 5 \times 10^{6}$ tetrahedrons were created. A visual comparison between the two different mesh densities is provided in the close-ups of figure 5 .

Numerical simulations based on the two meshes provided results in terms of macroscopic force and deformation differing by less than 1\%, indicating a sufficient level of mesh independence for the present purposes. Nevertheless, in a few cases convergence issues 
arose with the coarser mesh in presence of cohesive interface behavior - see section 4.2 -, likely due to an insufficiently smooth representation of the particles-matrix interfaces. For this reason, it was eventually decided to work with the finer mesh only, which was used to generate all the results presented in the remainder of this paper.

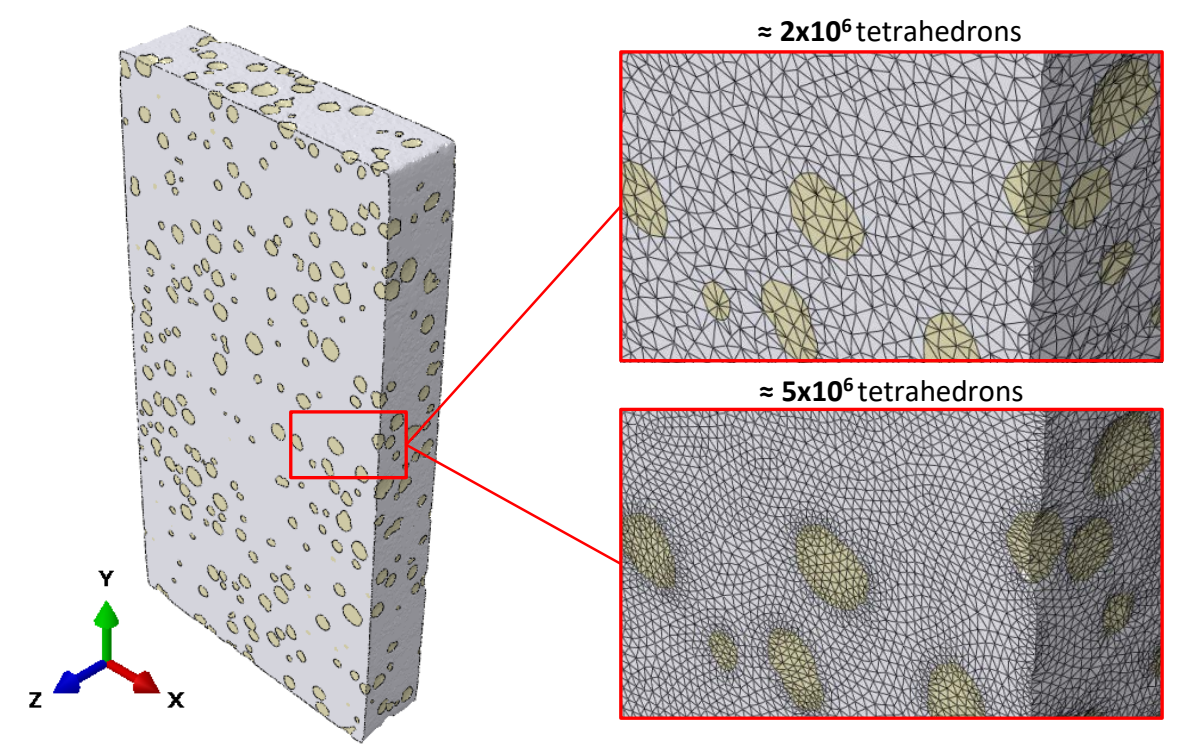

Figure 5 - Geometry of the full model representing the region highlighted in green between face $z^{+}$and $z^{-}$in figure 2 (a). The close-ups show the mesh densities correspoding to either $\approx 2 \times 10^{6}$ or $\approx 5 \times 10^{6}$ tetrahedrons.

\subsection{Mechanical behavior of the matrix-particles interface}

Direct measurements of the strength of the interface between the graphite particles and the matrix of DCI are not available in the literature. In general, there seems to be consensus among most of the models developed in the past on assuming the interface strength to be negligible (Andriollo et al., 2016a). This assumption is usually justified on the basis of microscopy observations of early debonding of particles sitting on the surface of tensile test specimens (Dong et al., 1997). On the other hand, as correctly pointed out by (Liu et al., 2002), the stress state around particles located in the bulk is likely different from that around particles sitting on the surface, therefore concluding that debonding occurs very early for all particles during tensile testing might be erroneous. 
In the present investigation, in order to avoid assuming arbitrary values of the interface strength, two extreme cases were considered: an interface with infinitely high strength and an interface with zero strength. For simplicity, these two will be referred to as "strong” and "weak" interfaces in the remainder of this work. Modelling the strong interface type in a finite element context is straightforward, as it is sufficient to make sure that the matrix and the particles share the same nodes at the boundary. Conversely, several possibilities exist for handling the weak interface. Here, the choice was the surface-based cohesive formulation available in ABAQUS (Dassault Systèmes Simulia Corp., 2017), which was the finite element software used in the present analyses. This formulation, which allows the specification of generalized traction-separation behavior between pairs of surfaces, was preferred over a standard contact formulation, as the latter would lead to an indetermination in the motion of the particles once they detach completely from the matrix. As only a continuously connected mesh could be generated in Avizo, a Matlab code was used to duplicate the nodes along the matrix-particle interfaces. While performing this operation, it was decided to leave particles formed by less than 50 elements - whose equivalent diameters lay in the range 10 to $20 \mu \mathrm{m}$ - connected to the matrix. Indeed, it was realized that the discretization of the interface of these particles was not smooth enough to guarantee convergence. Consequently, all weak interface simulations presented in this work were run considering - in practice - a strong interface between the matrix and those particles consisting of less than 50 elements. As figure 6 (a) shows, these represent $28 \%$ of the total 1544 particles discretized in the model, but account for only $1.7 \%$ of the total graphite volume. Therefore, this choice is not expected to affect the results significantly within the small range of deformation investigated. Concerning the type of traction-separation behavior, a linear elastic law with normal stiffness $k_{n n}$ and tangential stiffness $k_{n n} / 2$ was chosen. To ensure a practical fulfillment of the zero-strength interface condition, $k_{n n}$ was set to $10 \mathrm{MPa} / \mu \mathrm{m}$, which is a value sufficiently small to produce negligible effects on the macroscopic material response, see figure 6 (b). It is remarked that the normal stiffness quantified by $k_{n n}$ was set to apply only in presence of tension normal to the interface, whereas in presence of compression a hard contact behavior was enforced. 


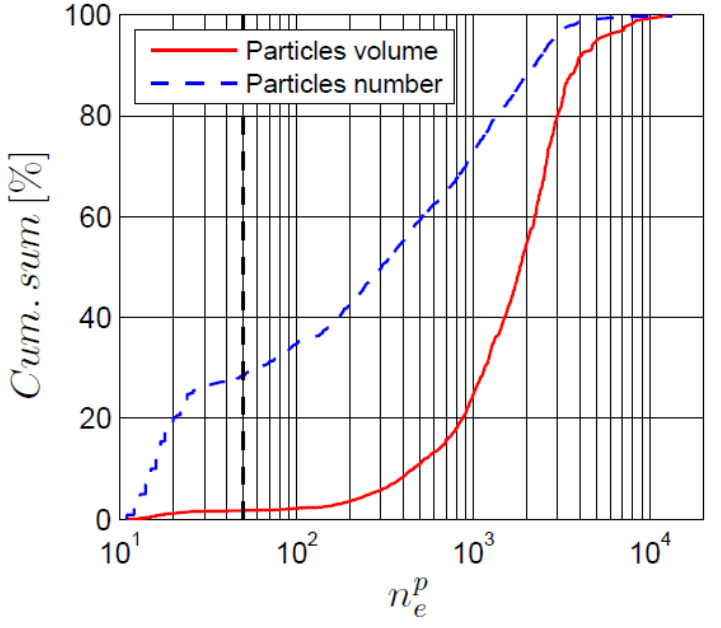

(a)

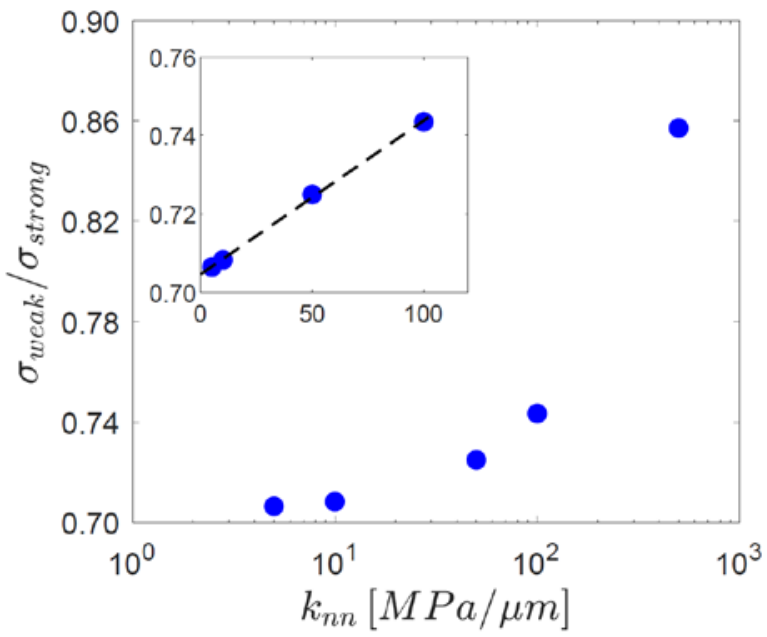

(b)

Figure 6 - Modeling the weak interface between the particles and the matrix. (a) Cumulative sum of the number and volume of the particles as a function of the number of elements $n_{e}^{p}$ inside a particle. The vertical dashed line indicate the threshold below which the cohesive formulation was not applied. (b) Tensile stress predicted by the finite element model at $3.87 \%$ uniaxial strain for different values of the cohesive elastic stiffness $k_{n n}$, normalized by the value corresponding to a strong interface.

\subsection{Mechanical behavior of the matrix}

To the authors' best knowledge, there is unanimous consensus in the DCI literature that the overall behavior of the matrix between the graphite particles can be described by standard isotropic constitutive theories, at least in those cases where the matrix is ferritic. Given the very low amount of pearlite and the random texture of the grains in the present DCI (Zhang et al., 2016), this was also the assumption made in the present work. More specifically, following (Andriollo et al., 2018b), it was assumed that the constitutive behavior of the matrix can be described by the elastic-visco-plastic model proposed by (Peric, 1993). The basic equations of the model are:

- Additive strain decomposition

$$
\boldsymbol{\varepsilon}^{t o t}=\boldsymbol{\varepsilon}^{e l}+\boldsymbol{\varepsilon}^{v p}+\boldsymbol{\varepsilon}^{t h}
$$

where $\boldsymbol{\varepsilon}^{t o t}, \boldsymbol{\varepsilon}^{e l}, \boldsymbol{\varepsilon}^{v p}, \boldsymbol{\varepsilon}^{\text {th }}$ denote the infinitesimal total, elastic, visco-plastic and thermal strain, respectively.

- Linear elastic law 


$$
\boldsymbol{\sigma}=\mathbb{C}^{e l}: \boldsymbol{\varepsilon}^{e l}
$$

where $\boldsymbol{\sigma}$ is the Cauchy stress and $\mathbb{C}^{e l}$ is the $4^{\text {th }}$ order isotropic elastic stiffness tensor.

- Von Mises-type yield function

$$
\Phi=\sigma_{e}(\boldsymbol{\sigma})-\sigma_{t h}\left(\varepsilon_{e q}^{v p}\right)
$$

where $\sigma_{e}$ is the von Mises stress, $\sigma_{t h}$ is the so-called stress threshold and $\varepsilon_{e q}^{v p}$ is the equivalent visco-plastic strain, which, for this particular model, can be defined via the evolution law $\dot{\varepsilon}_{e q}^{v p}=\dot{\lambda}$.

- Associative flow rule:

$$
\dot{\boldsymbol{\varepsilon}}^{v p}=\frac{\partial \Phi}{\partial \boldsymbol{\sigma}} \dot{\lambda}, \quad \dot{\lambda}= \begin{cases}\frac{1}{\xi}\left[\left(\frac{\sigma_{e}}{\sigma_{t h}}\right)^{1 / m}-1\right] & \text { if } \Phi\left(\boldsymbol{\sigma}, \sigma_{t h}\right)>0 \\ 0 & \text { if } \Phi\left(\boldsymbol{\sigma}, \sigma_{t h}\right) \leq 0\end{cases}
$$

where $m$ and $\xi$ are the strain-rate sensitivity and the viscosity-related parameter, respectively.

To complete the model, the Voce-type strain hardening law

$$
\sigma_{t h}\left(\varepsilon_{e q}^{v p}\right)=R_{0}+\left(R_{\infty}-R_{0}\right) \times\left(1-\exp \left(-\varepsilon_{e q}^{v p} / \varepsilon^{*}\right)\right)
$$

was selected, where $R_{0}, R_{\infty}$ and $\varepsilon^{*}$ are material parameters.

The main reason for choosing Peric's model is its behavior in the limit $\sigma_{t h} \rightarrow 0$ and $\xi \rightarrow 0$. Indeed, in the first case the model reduces to the well-known Norton-Hoff model, which is used to describe power-law creep at high temperature. In the second case, the model instead reduces to the standard rate-independent J2-flow theory of plasticity, which is normally adopted to describe the behavior of metals at room temperature. In such a condition, $\sigma_{t h}$ defines the yield stress evolution, with $R_{0}$ and $R_{\infty}$ representing the initial yield stress and the ultimate tensile strength respectively. As a consequence, Peric's model is well suited for the present investigation, where applicability during both the cooling stage of the manufacturing process and the room temperature tensile loading is required.

Peric's model was implemented as a UMAT subroutine in ABAQUS, following the implicit discretization scheme and integration algorithm proposed in (de Souza Neto et al., 2008). Values of the model parameters were taken from (Andriollo et al., 2018c), where, as already mentioned in section 2, a material equivalent to the DCI matrix was manufactured 
and tested at various temperatures. Concerning this, an indication of the reliability of the experimental data of (Andriollo et al., 2018b) can be obtained by comparing it to the findings of (Kuna and Sun, 1996). Indeed, the latter authors followed an almost analogous strategy to obtain a room temperature tensile curve for the matrix of the same grade of DCI used in the present investigation. The only difference is that a rolling operation was additionally performed to adjust the grain size of the equivalent material to that of the DCI matrix, while in (Andriollo et al., 2018c) expressions available in the literature were used to compensate for the effect of this parameter. Remarkably, figure 7 indicates that the two sets of tensile data agree fairly well. It is worth pointing out that a tensile curve for the matrix of this grade of DCI was also provided by (Zhang et al., 1999). Nevertheless, as figure 7 shows, the corresponding stress lies constantly 50-80 MPa below that reported by the two previous groups of researchers. As no information is given in (Zhang et al., 1999) concerning how the tensile data was obtained, it is not possible to analyze the reasons for this deviation.

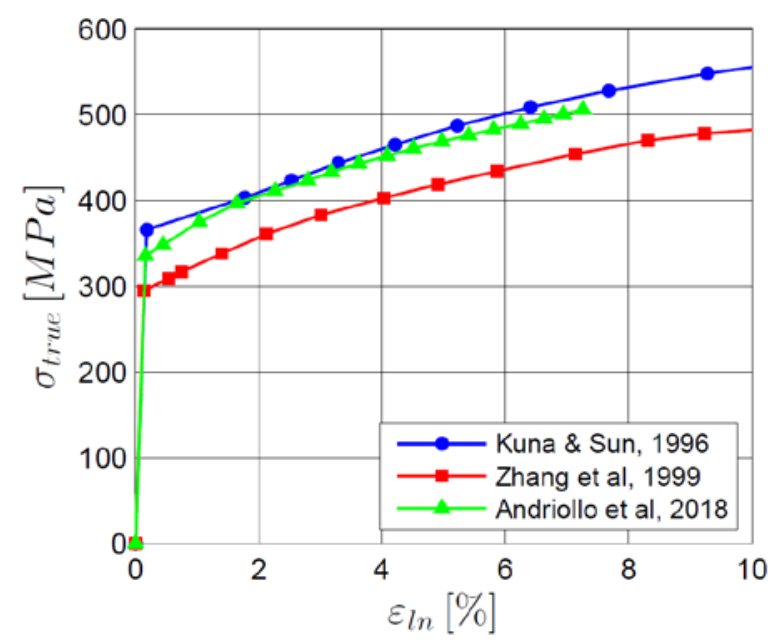

Figure 7 - Room-temperature tensile data available in the literature for the matrix of the DCI grade considered in the present investigation.

\subsection{Mechanical behavior of the particles}

Four different possibilities to model the graphite particles were investigated in the present work. In a first step, the particles were assimilated to voids. From a practical point of view, this was achieved by modelling them as elastic bodies with Young's modulus equal to 1 
MPa. In a second step, similarly to what done in most of the previous studies of DCI, the particles were assumed to deform according to an isotropic, linear thermo-elastic law. Young's modulus and Poisson's ratio were set to $15 \mathrm{GPa}$ and 0.3, respectively, as these values are consistent with the macroscopic elastic behavior of DCI - according to unit cell models, see the discussion in (Andriollo and Hattel, 2016) - and are also in agreement with the average elastic response of the particles measured via nano-indentation (Andriollo et al., 2018a). The thermal expansion coefficient of isotropic fine-grain graphite IGI-110 was assumed to apply (Tsang et al., 2005). In a third step, the particles were still modelled as homogeneous and isotropic, but yielding was allowed according to the standard J2-flow theory of plasticity. A constant yield stress of $25 \mathrm{MPa}$ was chosen, which is typical of bulk isotropic graphite (Bonora and Ruggiero, 2005). Finally, in a fourth step the particles were assumed to deform elastically, but their highly anisotropic, inhomogeneous internal structure was modelled in an explicit way. The reasons for considering this option in spite of its high computational cost and the details of the implementation are given in the two following sub-sections.

\subsubsection{Link between structure and properties}

Thorough electron microscopy investigations (Ghassemali et al., 2019; Miao et al., 1994, 1990; Monchoux et al., 2001; Theuwissen et al., 2016, 2014, 2012) have clarified that each graphite particle consists of conical sectors radiating from the particle's center to the outer periphery. The sectors are in turn composed of graphite platelets oriented perpendicularly to the radial direction, with each platelet consisting of a stack of graphene layers. Some studies have indicated that the conical sectors do not reach the particle's surface: indeed, a thin superficial layer formed by smaller and highly misoriented graphite crystals exists (Monchoux et al., 2001). A qualitative visual confirmation of the subdivision into conical sectors and superficial layer of one particle belonging to the present DCI is provided in figure 8 (a). On the same figure, the expected further subdivision of one sector into graphite platelets is indicated.

The anisotropic nature of the platelets - which stems from the anisotropy of the graphene layers - combined with their non-uniform arrangement, implies that the properties of each 
particle are both spatial and orientation dependent. A clear indication of this has recently been obtained via nano-indentation by showing that the resistance against penetration correlates well with the expected variation of the platelets' orientation within the particle (Andriollo et al., 2018a). Furthermore, as mentioned in the introduction, the abrupt change in the platelets' orientation at the boundaries of the conical sectors is believed to be the cause of the large perturbations of the residual elastic field detected in proximity to the

particles (Andriollo et al., 2018b; Zhang et al., 2019). These findings suggest that the internal structure of the particles might have an influence on the DCI behavior during loading, either directly - by exhibiting different stiffness and strength according to the load type and direction - or indirectly - by generating residual stress concentrations in the matrix leading to e.g. its premature yielding.

\subsubsection{Implementation of an anisotropic thermo-elastic model}

In this work the particles' internal structure was taken into account following the approach introduced in (Andriollo et al., 2016a, 2016b), which allowed reproducing the complex local residual stress field existing around the single particle considered in (Andriollo et al., 2018b). As the real structure is not accessible with non-destructive methods, the basic idea is to subdivide the volume of a particle into a realistic number of conical sectors according to the idealized geometry of figure 8 (b). Subsequently, the particle is meshed and anisotropic properties are assigned to each element depending on the orientation of the sector it belongs to. This procedure, however, had to be slightly modified in the present case as a sector-conforming mesh could not be created for each of the 1544 particles contained in the model. Therefore, the original mesh generated by Avizo without considering any internal subdivisions of the particles was retained. Subsequently, the idealized geometry of figure 8 (b) was superimposed to each particle, with the centroid of the former coinciding with the center of mass of the latter. Finally, each particle element was assigned to one of the 80 sectors of the idealized geometry on the basis of its position. As an example, figure 8 (c) shows in different colors the elements assigned to the 10 sectors associated with positive values of the local coordinates $[\mathrm{r}, \mathrm{s}, \mathrm{t}]$ of figure 8 (b). 
A similar strategy was also used to identify the elements forming the superficial layer. In this case, an element was classified as belonging to the former if its centroid was located at a distance from the matrix less than $5 \%$ of the equivalent particle radius. Moreover, all elements having one or more faces on the particle boundary were also assigned to the surface layer, in order to avoid particles made of a few elements only to remain without surface layer. The ratio between the volume occupied by all the elements assigned to the surface layer and the total volume of the particles turned out to be equivalent to that of a single spherical particle having a surface layer with thickness $10.1 \%$ of its radius, which is a value very close to those reported in the literature for real particles (Monchoux et al., 2001).

Following (Andriollo et al., 2016b), the mechanical behavior of both conical sectors and superficial layer was assumed to be linear thermo-elastic. More specifically, each conical sector was assumed to have the elastic symmetry characteristic of rhombohedral graphite, with the plane of isotropy oriented perpendicularly to the radial axis passing through the sector centroid. To account for the decrease in stiffness due to the presence of defects such as twins and bending planes within the platelets, the elastic moduli were assumed to be those valid for a perfect stack of graphene layers, but multiplied by a factor 0.15 . As demonstrated in (Andriollo et al., 2018b) and supported by the findings of (Qin et al., 2016), this choice allows for a precise prediction of the local residual stresses in the neighboring matrix. The superficial layer of the particles was assumed to be isotropic instead, with Young's modulus and Poisson's ratio equal to $10 \mathrm{GPa}$ and 0.2 respectively, for the reasons discussed in (Andriollo et al., 2016b). No temperature dependence was taken into account, as this quantity seems to affect the elastic properties of graphite only to a limited extent within the temperature range considered (Faris et al., 1952). Concerning the thermal contraction, the values of graphene were employed for the conical sectors, whereas the values of the isotropic fine-grain graphite IGI-110 were used for the superficial layer. Both sets of data were taken from (Tsang et al., 2005). 


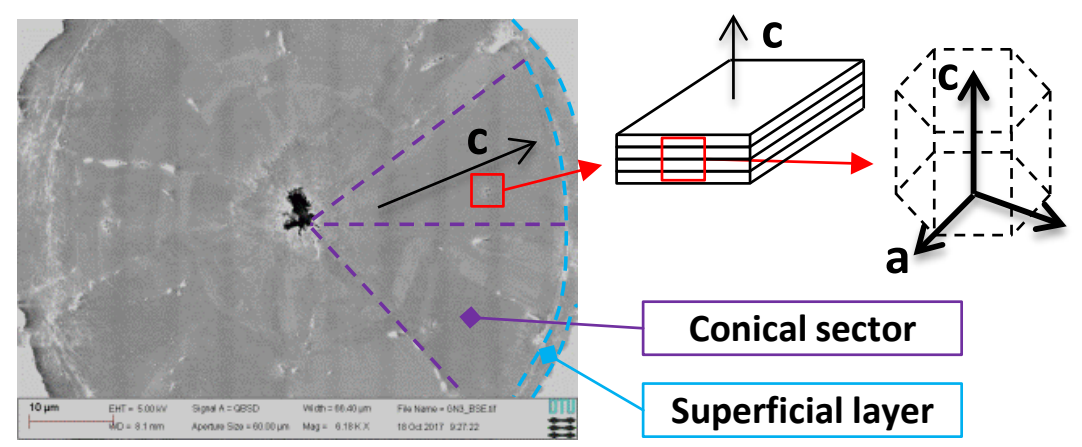

(a)

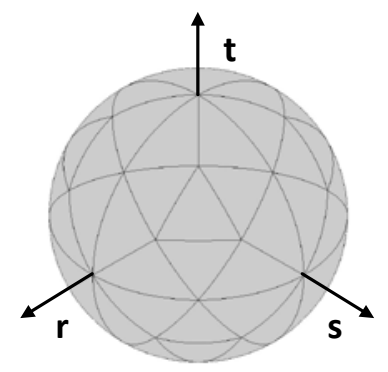

(b)

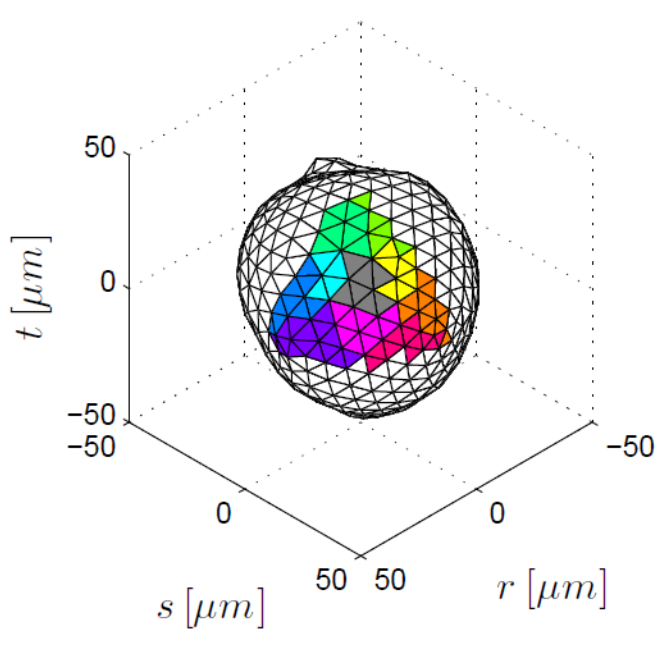

(c)

Figure 8 - Modeling the anisotropic internal structure of the particles. (a) Scanning electron microscopy image of a particle extracted from the DCI used in this study, showing the division into conical sectors and the presence of the surface layer. The expected orientation of the graphite platelets is also indicated. (b) Idealized 3D geometrical model of the division of a particle into sectors. (c) Elements forming a particle colored according to the different sectors of the geometrical model in (b) they belong to.

\subsection{Boundary conditions}

\subsubsection{Formation of micro-scale residual stresses}

The formation of micro-scale residual stresses during manufacturing was simulated by prescribing a uniform temperature variation to the model according to the cooling curve reported in (Andriollo et al., 2018b), which relates to the precise location in the large DCI component from which the tensile specimen used in the present investigation was extracted. In contrast to what was done in (Andriollo et al., 2018c), cooling was started from a 
temperature just below the end of the eutectoid reaction, corresponding to $792{ }^{\circ} \mathrm{C}$. Indeed, phenomena occurring above this point have a minor impact on the final residual stress state (Andriollo et al., 2018b) and, in addition, the increase in dislocation density produced during the eutectoid reaction does not affect the matrix yield stress at room temperature significantly (Zhang et al., 2019). During cooling, as no information is available for the displacement of the microstructure, a zero surface traction condition was applied to all model boundaries, i.e. the $z^{+}, z^{-}$and lateral faces. Admittedly, this might be a source of error, even though it is consistent with the fact that once the specimen is extracted from the DCI component any force acting on its lateral faces is released.

\subsubsection{Tensile testing}

To simulate the tensile test, the microscopic displacement calculated via DVC was prescribed on the $z^{+}$and $z^{-}$faces of the full model. By contrast, the zero surface traction condition was maintained on the lateral faces, as they are part of the free surface of the specimen. The displacement associated with the combined increment $1 \& 2$ was prescribed first, followed by that associated with increment 3 . In this respect, it is noted that the nodal density over the $z^{+}$and $z^{-}$faces is much larger than the density of points at which the displacement is known, which correspond to the centers of the DVC subvolumes. Consequently, the displacement of each finite element node was interpolated on the basis of that of the four closest subvolumes by means of the standard shape functions of 2D quadrilateral elements.

A special comment has to be made concerning which displacement components (DCs) were prescribed. Indeed, as all the DCs are known from DVC, the displacement could be enforced along all directions. On the other hand, as the DCs are available only after relatively large increments, during which the material behavior can change substantially, the question of how the DCs are to be set to vary within each increment arises. If a linear variation of each DC is assumed, meaning that the DCs are related to each other by linear functions, spurious stresses may arise which alter significantly the shape of the uniaxial stress vs. strain curve. This effect is demonstrated in figure 9 (a), where the stress vs. strain response recorded during straining of an infinitesimal matrix element is shown assuming 
that either 1) the element can contract freely in the lateral direction, or that 2) the lateral contraction is set to vary linearly between the initial and final values recorded in case 1). It can be observed that, despite the final stress values coincide, the apparent stiffness and yield stress in case 2 ) are $\approx 9 \%$ and $20 \%$ lower than in case 1 ). This indicates that if all DCs were prescribed in the present model, the predicted tensile stress during the elastoplastic transition would probably be biased by the spurious stresses arising from the fact that, in the real tensile test, the lateral contraction of the volume $\mathrm{V}$ does not vary linearly with its extension along the z-axis. This speculation is supported by the close similarities between the curves of figure 9 (a) and those of figure 9 (b), which represent the macroscopic response of volume $\mathrm{V}$ predicted when either all or only the z-component of the displacement is prescribed. As a prediction of the tensile stress as accurate as possible over the entire elastic-plastic transition is sought in this work, the choice was then to prescribe only the z-component of the displacement, leaving the material free to contract laterally according to the constitutive model.

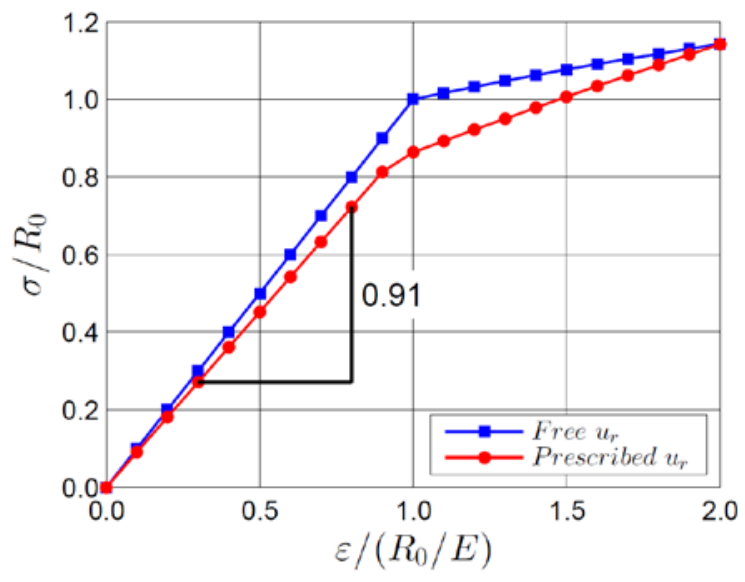

(a)

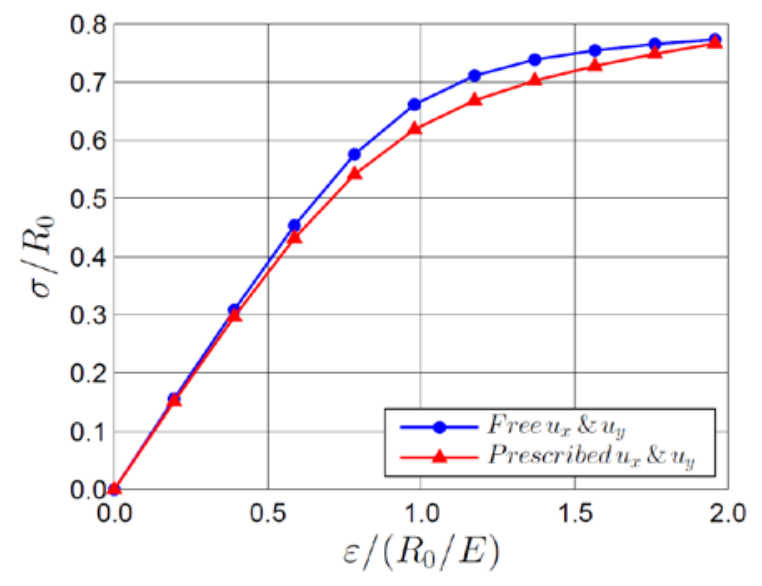

(b)

Figure 9 - Bias in the prediction of the apparent elastic stiffness and yield stress due to the linear approximation used to prescribe the lateral contraction. (a) Stress vs. strain response recorded during straining of an infinitesimal matrix element assuming that either 1) the element can contract freely in the lateral direction, or that 2) the lateral contraction is prescribed to vary linearly between the initial and final values recorded in case 1). (b) Stress vs. strain response predicted by the full model during increment $1 \& 2$ when either all or only the z-component of the displacement is prescribed. The data refers to a simulation run considering a weak interface, isotropic elastic behavior of the particles and neglecting the residual stresses. 


\section{Results and discussion}

In this section, the outcome of the full model is compared first to the macro-scale data recorded during the tensile test, then to the micro-scale strain reconstructed with DVC and finally to the macro-scale predictions of the cubic unit cell model. For each type of comparison, eleven numerical simulations are considered. Eight correspond to selecting different options for the initial stress state (with / without micro-scale residual stresses (RS)), the interface strength (strong / weak) and the elastic behavior of the particles (isotropic, if the internal structure is neglected / anisotropic, if it is accounted for). Two are meant to show the effect of isotropic plasticity in the graphite particles, assuming a weak interface. Finally, one relates to the special case in which the particles are considered as voids, for which the concepts of interface strength and micro-scale residual stress become inapplicable. Each simulation was run in parallel on 20 cores of a Linux 6.4 cluster based on Intel Xeon Processor X5550, and took between 10 and 20 hours depending on whether the manufacturing process was simulated or not.

\subsection{Full model vs. experiments: Macroscale comparison}

\subsubsection{Yielding behavior}

As already mentioned in section 3.1, the end of the second strain increment applied during the in-situ test corresponds, approximately, to the macroscopic yield point of the material. The evolution of the macroscopic tensile stress as predicted by the model up to this level of deformation is depicted in figure 10. Values for 5 out of 11 simulations are considered, corresponding to increasing levels of complexity of the model. It can be seen that the spread in the model predictions, associated with different choices of the simulation parameters, tend to widen as the deformation progresses. In this respect, some of the simulations capture quite well the stress measured at the end of the increment, while others overestimate this quantity significantly. To provide additional insight into this aspect, the relative error is reported in figure 11 for all the 11 simulations. The error on the average lateral contraction of volume $\mathrm{V}$ along the $\mathrm{y}$-axis - see figure 5 - is shown as well, as 
significant deviations from the experimental value of $0.094 \%$ - determined via DVC might exist due to the fact that only the z-component of the displacement was prescribed over the $z^{+}$and $z^{-}$faces of the model. The figure shows that both types of error are always below $25 \%$ and, in particular, are as low as $5 \%$ when the particles are modelled as elastic anisotropic bodies, a weak interface is assumed to exist and the residual stresses are accounted for. It is worth emphasizing that errors of a few percent can be justified based on the uncertainty affecting the DVC procedure as well as the constitutive behavior of the matrix and the graphite particles - see the discussion in sections 3.2, 4.3 and 4.4.

At this point, two observations can be made. First, from a more general perspective, the very good agreement between model predictions and measurements constitutes the proof that the present experimental-computational approach is viable. Second, the simulation that provides the smallest errors assumes that rupture of the interface takes place before macroscopic yielding, as suggested in the studies discussed in section 4.2. Hence, the present results support this hypothesis, even though they rule out the possibility that the particles can, as a consequence, be approximated to voids. Indeed, significantly larger errors of $10 \%$ and $17 \%$ are recorded for the corresponding simulation, likely due to the fact that the particles, despite weakly bonded to the matrix, can still carry compressive forces that 1) enable the formation of residual stresses and 2) oppose the lateral contraction of the matrix during tensile loading, as pointed out by (Bonora and Ruggiero, 2005).

The results of figure 10 can also be used to attempt an analysis of the mechanisms controlling the plastic deformation of the material at very small strains. Indeed, it is a welldocumented fact that no cast iron grade exhibits perfect linear elasticity. Normally, this is justified on the basis of the stress concentration induced in the matrix by empty cavities having the shape of the graphitic phase. With respect to DCI, deviations from linear stressstrain dependence were observed at stresses of only a fraction of the yield stress by means of highly-accurate electric resistance strain gauges (Kohout, 2001). Furthermore, inelastic deformation occurring in the seemingly elastic deformation range was also detected via acoustic emission (Sjögren and Svensson, 2005). In contrast to other grades of cast iron, though, the hypothetic stress concentration in DCI is much lower, due to the near spherical shape of the graphite particles, and it does not seem sufficient to explain the very early 
onset of the inelastic deformation (Kohout, 2001). To shed light on this topic, the derivative of the macroscopic tensile curves of figure 10 is plotted in figure 12. On the same figure, the data of (Kohout, 2001), which is up to $0.1 \%$ strain and refers to a DCI with almost the same chemical composition, is reported as well. From the experimental data, it is seen that the material instantaneous stiffness reduces by as much as $20 \mathrm{MPa}$ within the first $0.05 \%$ straining. According to the model, this effect is not justifiable by assuming that the particles correspond to voids, hence corroborating the conclusion of (Kohout, 2001). On the other hand, when the weak interface and the residual stresses are taken into account, the relative reduction in stiffness becomes very close to the measured one, even though the absolute values are overestimated by $\approx 10 \mathrm{MPa}$. Remarkably, this overestimation disappears if the anisotropic internal structure of the particles is also included in the simulation. Moreover, if the experimental stress and strain values are normalized according to the ratio between the yield stress of the material used in (Kohout, 2001) - $270 \mathrm{MPa}$ - and the one of the present DCI - $235 \mathrm{MPa}$-, an almost perfect overlapping of the two curves is obtained over the entire strain range. These findings indicate that, as partially hypothesized in section 4.4.1, the complex residual stress field generated by the interaction between the matrix and the highly anisotropic structure of the particles has a significant impact on the DCI response at small strains. 


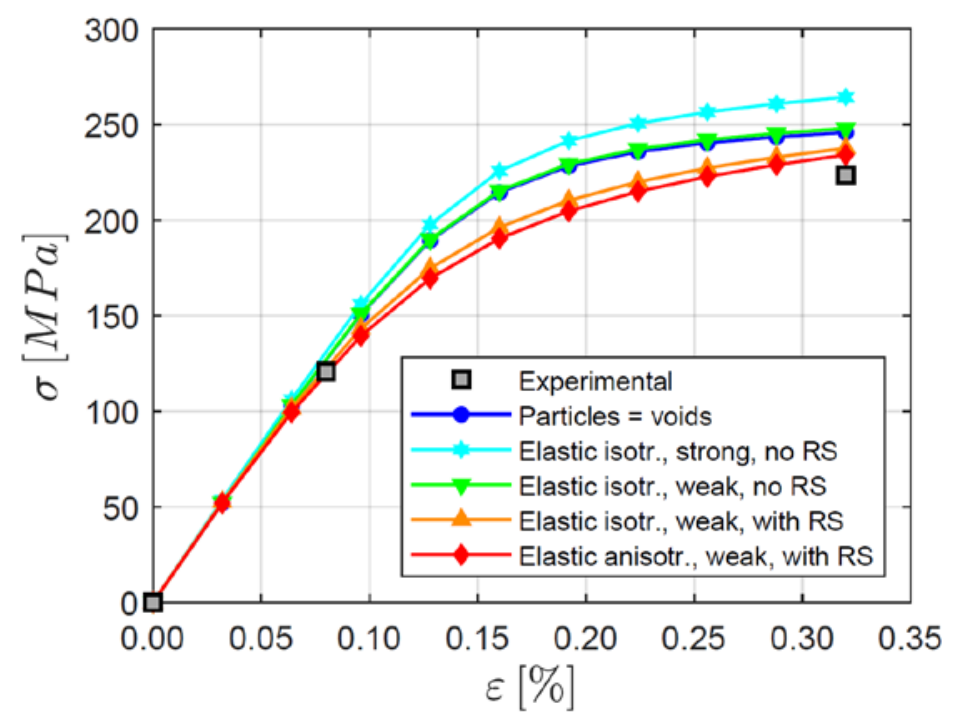

Figure 10 - Tensile stress predicted by the full model during the combined increment $1 \& 2$, for simulations with increasing level of complexity (the assumptions made for the mechanical behavior of the particles, the interface type and the initial stress state are indicated in the legend). The experimental data measured during the in-situ tensile test is also reported.

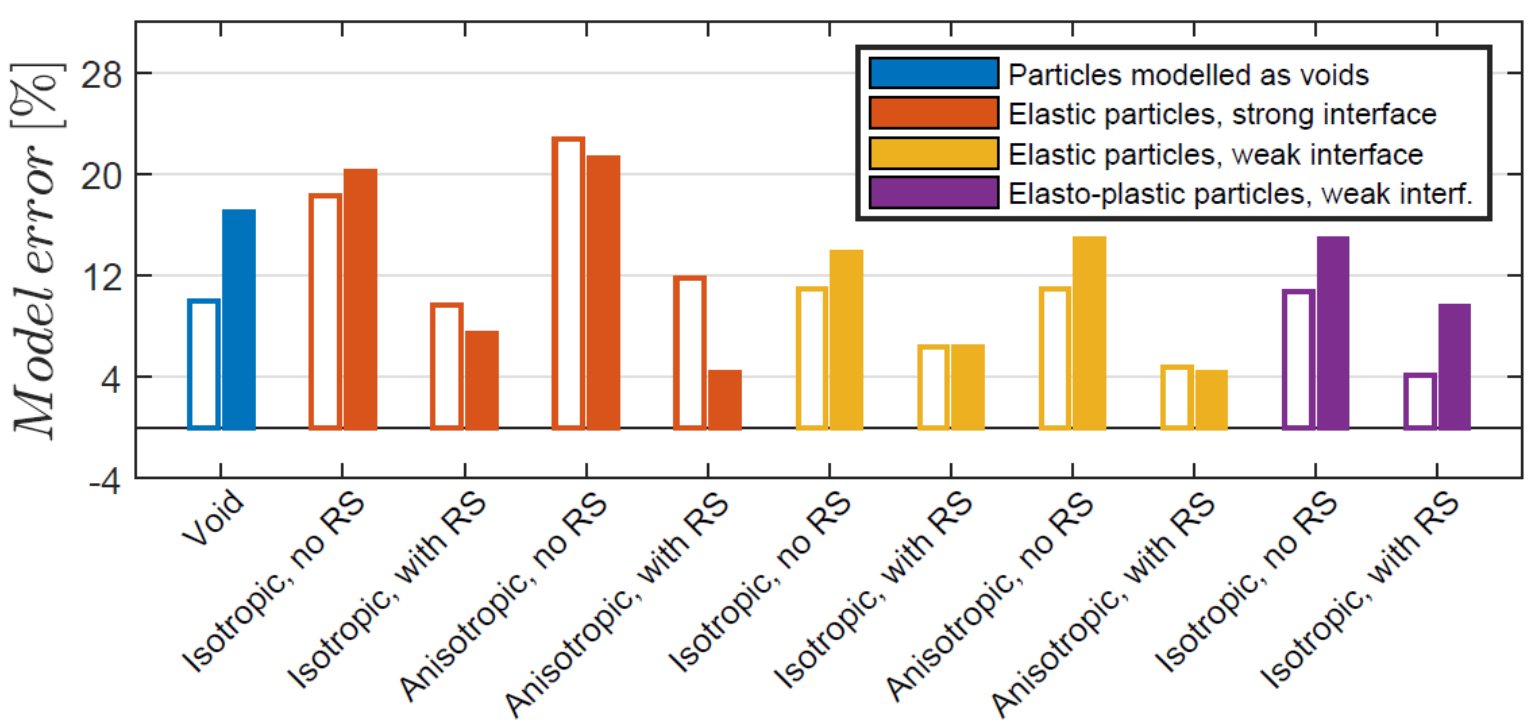

Figure 11 - Relative error of the full model in terms of tensile stress (empty bars) and lateral contraction of the specimen (full bars) at the end of the combined increment $1 \& 2$. A positive error indicates that the quantity is overpredicted, a negative error that the quantity is underpredicted. 


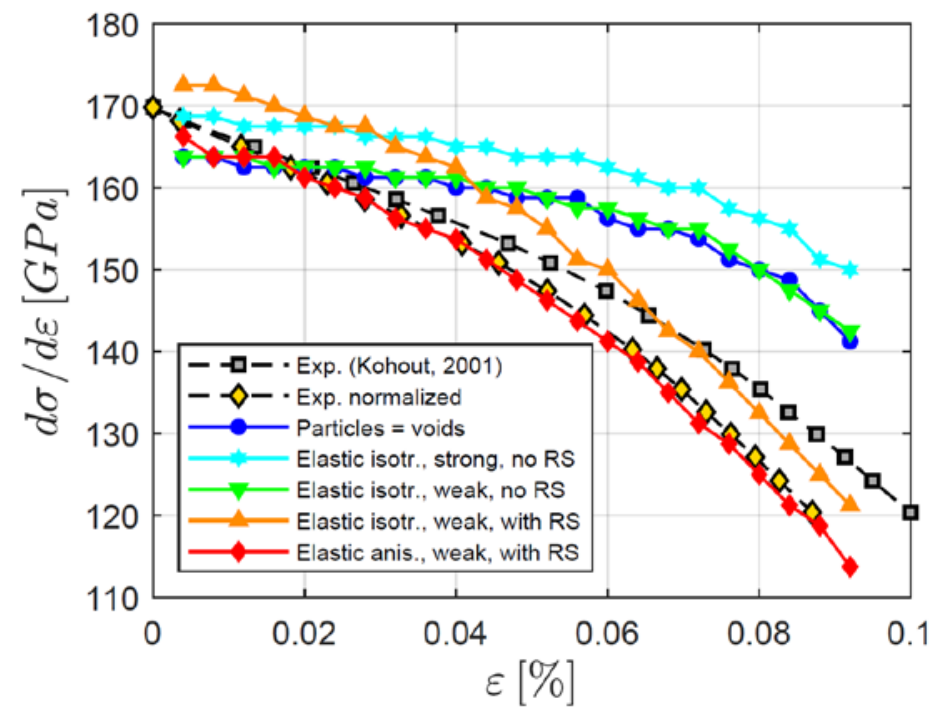

Figure 12 - Derivative of the numerical tensile curves of figure 10 at very small strains. The experimental data of (Kohout, 2001) is reported for comparison, in both the original (unmodified) and the normalized form. The normalized form corresponds to normalizing the experimental stress and strain by the ratio between the yield stress of the material used in (Kohout, 2001) and that of the DCI employed in the present investigation.

\subsubsection{Hardening behavior}

The magnitude of the third and final strain increment applied to the specimen was adjusted to produce extensive plastic deformation, in order to investigate the hardening behavior of the material. The evolution of the macroscopic stress as predicted by the model up to the corresponding strain level is shown in figure 13. A small kink in the curves can be seen at $0.32 \%$ deformation, which marks the end of the combined increment $1 \& 2$ and the beginning of increment 3 . The reason is the change in the shape of the displacement pattern prescribed as boundary condition, which drives the deformation of the model. Smaller increments would probably minimize this effect. Figure 14 reports the model errors in terms of stress and lateral contraction with respect to the corresponding experimental values of 348.9 MPa and $1.38 \%$, as determined at the end of increment 3. To discuss the influence of the simulation parameters, it is convenient to focus on the assumption made for the interface behavior, as its impact on the results seems considerably greater than that of the residual stresses and of the particles' mechanical behavior, at least when the latter is 
assumed elastic. Indeed, both the error on the stress and that on the lateral contraction are greater than $20 \%$ when a strong interface is assumed, whereas they are consistently in the order of 8 - $12 \%$ when a weak interface is considered. The large overestimation in the former case is due to the fact that, at this level of deformation, the interface is likely broken over a large portion of the particles' surface, as complete debonding is expected before macroscopic fracture of the material (Hütter et al., 2015). In this respect, it is worth pointing out that the stress overestimation is at a minimum when either the particles are assimilated to voids or they are assumed to deform plastically. The reason is that, as mentioned previously, the particles can continue hindering the lateral contraction of the matrix even after debonding has occurred at the interface locations perpendicular to the direction of macroscopic straining. According to the simulations run considering a weak interface and elastic behavior of the particles, the compressive stresses over the portion of the particles' surface parallel to the straining direction are, on average, as high as $\approx 300$ MPa. These very high values, which are a consequence of the purely elastic model used to describe the particles, are probably unrealistic. Indeed, once the particles are not confined by the matrix in all directions anymore, due to partial debonding, they will likely deform plastically at some point to fill the empty volume. It is worth emphasizing, though, that when the load carrying capacity of the particles is decreased, the matrix can contract more, and this increases the overestimation of the lateral contraction. Indeed, figure 14 shows that the corresponding error is of $\approx 10 \%$ for the simulations run considering a weak interface and elastic behavior, whereas it is $\approx 17 \%$ when the particles are either assimilated to voids or allowed to deform plastically. This suggests that error sources other than the particles' behavior come into play at this relatively large level of deformation. 


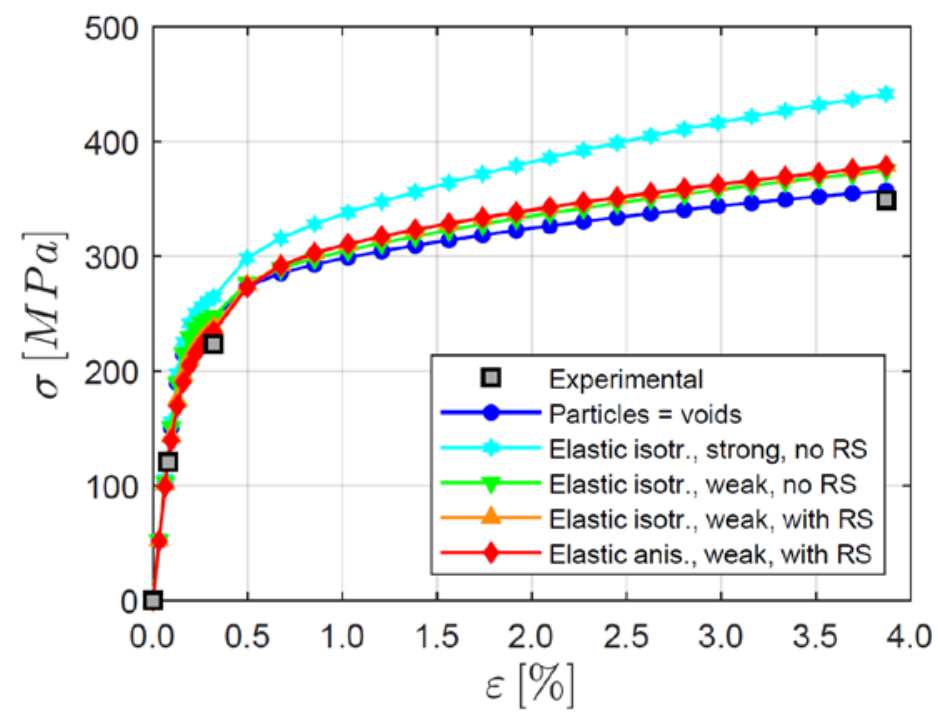

Figure 13 - Tensile stress predicted by the model during both the combined increment $1 \& 2$ and increment 3 , for simulations with increasing level of complexity (the assumptions made for the mechanical behavior of the particles, the interface type and the initial stress state are indicated in the legend). The experimental data measured during the in-situ tensile test is also reported.

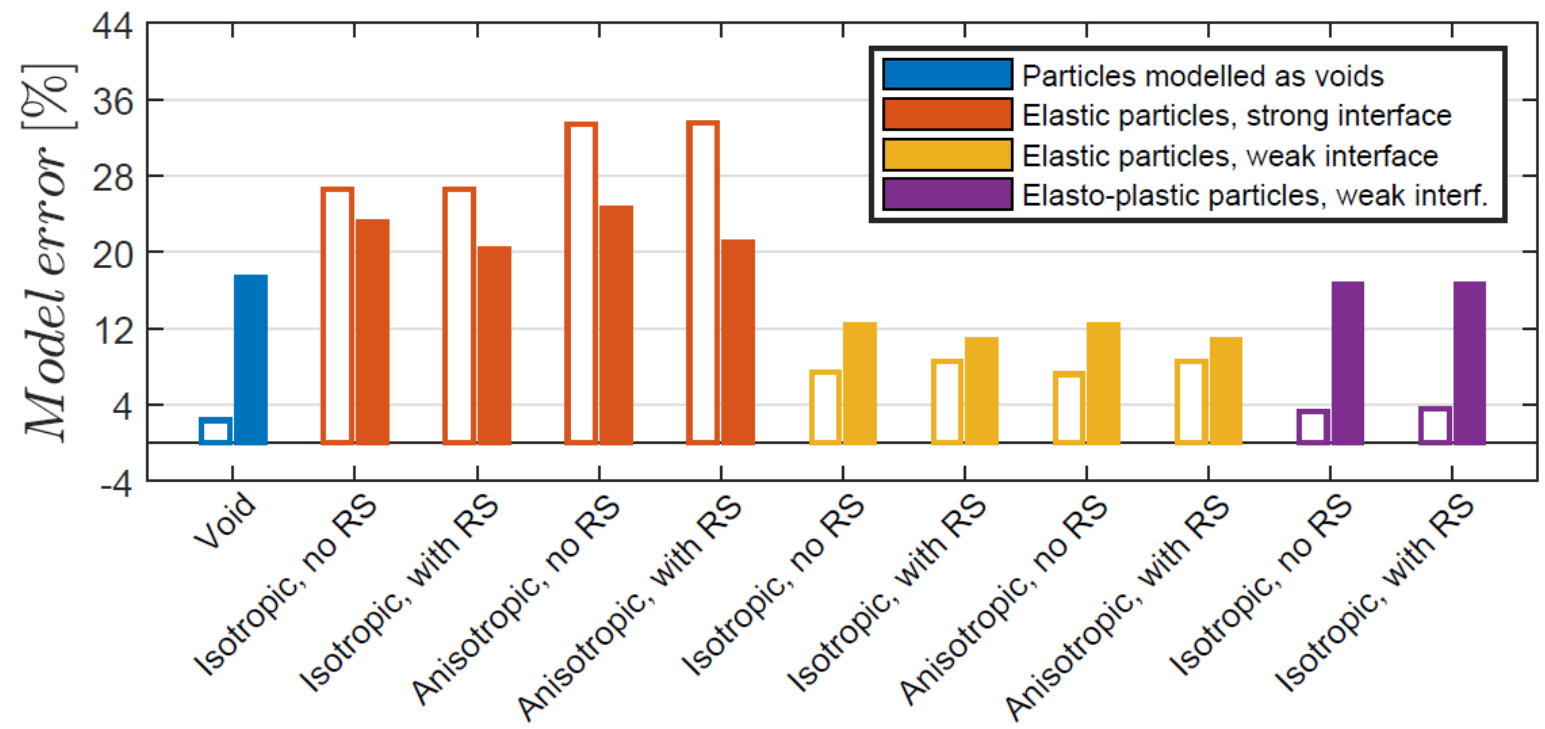

Figure 14 - Relative error of the full model in terms of tensile stress (empty bars) and lateral contraction of the specimen (full bars) at the end of increment 3. A positive error indicates that the quantity is overpredicted, a negative error that the quantity is underpredicted. 


\subsection{Full model vs. experiments: Microscale comparison}

The comparison between model predictions and DVC data is not straightforward when a subset-based DVC algorithm is employed, due to the different locations at which the displacement vector is known. In the present case, as the finite element mesh is much finer than the grid used in the DVC procedure, it was decided to make the comparison at the level of the DVC grid. Accordingly, the displacement of each subvolume, as predicted by the model, was considered equal to the mean displacement of all the finite element nodes located inside that subvolume. To enable the comparison in terms of a quantity related to the material deformation, a new grid translated by $1 / 2$ of the size of the subvolumes along all the three axes was introduced. Then, at each grid point, the equivalent strain $\varepsilon_{e q}=$ $\left(2 \varepsilon_{i j} \varepsilon_{i j} / 3\right)^{0.5}$ was calculated from the displacement at the centers of the eight neighboring subvolumes by means of the shape functions of the standard 8-node tetrahedron.

Figure 15 (a) and (b) compare the equivalent strain based on the DVC displacement with that based on the displacement predicted by the model, for the combined increment $1 \& 2$. The region chosen for the comparison is the specimen's cross-section located mid-way between the $z^{-}$and the $z^{+}$face, which is the most distant from the model boundaries where the displacement is prescribed. The contours show that non-negligible differences exist between the two sets of data, despite the fact that the one associated with the model relates to the simulation that gives the lowest error at the macro-scale, i.e. the one where the particles are considered elastic anisotropic, a weak interface is assumed and the micro-scale residual stresses are accounted for. To quantify the differences the following deviation parameter is introduced:

$$
\text { Deviation }=\sqrt{\frac{\sum_{k}\left(\varepsilon_{e q, k}^{D V C}-\varepsilon_{e q, k}^{F E}\right)^{2}}{\sum_{k}\left(\varepsilon_{e q, k}^{D V C}\right)^{2}}}
$$

where the superscripts DVC and FE refer to DVC-based and model-based data, respectively, and the summation spans all the grid points located on the cross-section. According to this formula, the deviation is as large as $44 \%$, and varies by no more than +1 $\%$ if the outcome of the other simulations is considered for the comparison. Negligible 
changes are also recorded when the simulations are repeated prescribing all the displacement components on the $z^{-}$and the $z^{+}$face of the model, instead of just the one along the z-axis. Figure 15 (c) shows that the two sets of data differ mainly near the edges of the cross-section, close to the locations where odd values of the displacement field were recorded with DVC - see figure 4 (b) and (c) and the related discussion in the text. In all the other areas, the strain difference does not exceed $0.2 \%$ and, therefore, it can be explained by the noise affecting the DVC data. Indeed, with respect to the same crosssection, the equivalent strain associated with the two CT maps of the specimen in the unloaded configuration - which should vanish, as discussed in section 3.2.1 - has an average value of $0.16 \%$ and a peak of $0.26 \%$.

The situation changes completely when the third increment is considered, i.e. after significant plastic deformation of the specimen. Indeed, figure 16 shows that in this case the equivalent strain contour reconstructed via DVC agrees very well with that predicted by the model, except for a tiny area close to the top edge of the cross-section. Accordingly, figure 17 indicates that the deviation calculated with equation (12) for all the 11 simulations is much smaller, ranging from $8 \%$ to $13 \%$. Concerning this, the pattern of figure 17 resembles very much that of the error on the specimen's lateral contraction seen in figure 14, meaning that the macro-scale and the micro-scale comparisons are consistent with each other. Thus, it can be concluded that the factors responsible for the differences between the equivalent strain contours of figure 15 (a) and (b) become unimportant when DCI is strained beyond its macroscopic yield point. This supports the hypothesis that the former deviation is mainly an artifact of the insufficient resolution of the DVC procedure, and vanishes at a larger deformation because the latter improves as discussed in section 3.2.1. As a final remark, it is worth pointing out that the results of figure 16 and figure 17 suggest that the model assumption of neglecting both the anisotropy of the individual grains of the matrix as well as the heterogeneity associated with the small amount of pearlite is reasonable within the context of the present analysis. 


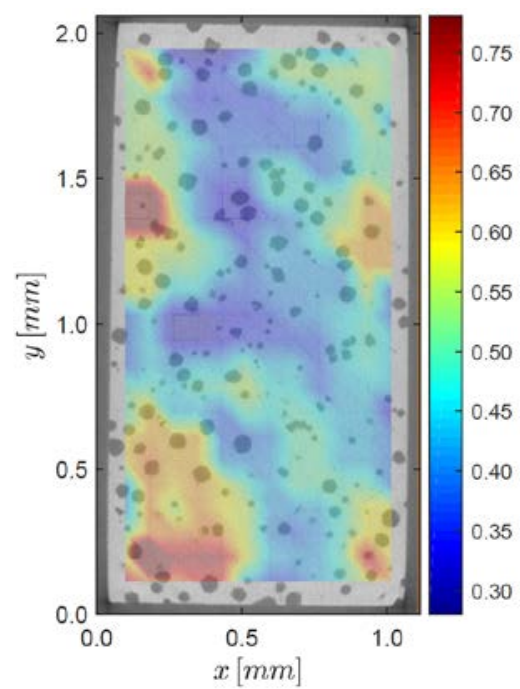

(a)

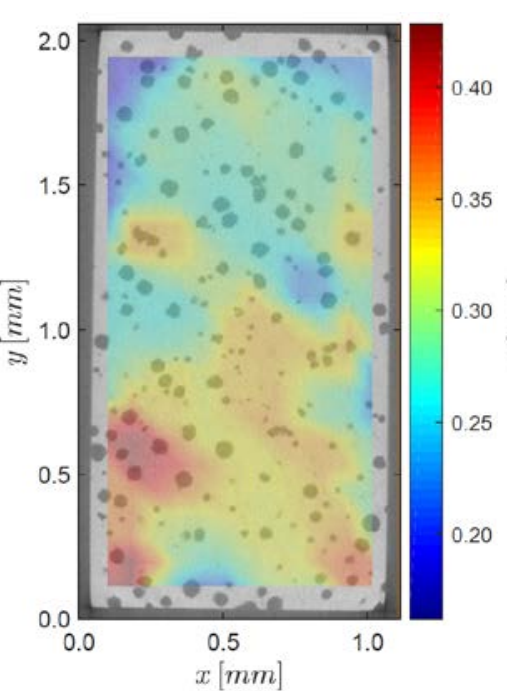

(b)

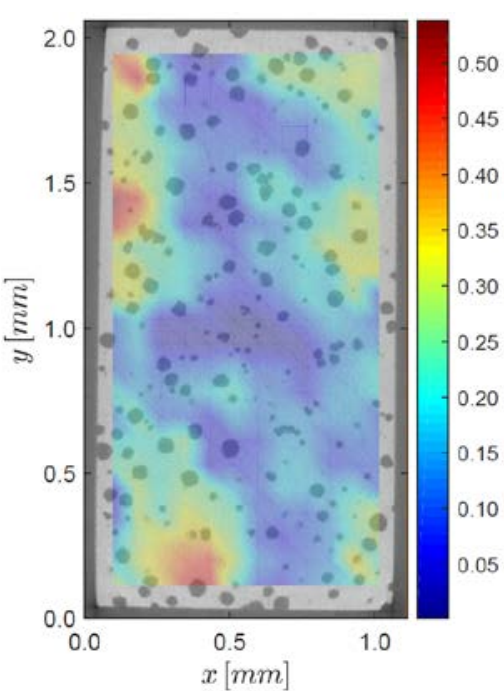

(c)

Figure 15 - Contour of the equivalent strain (in \%) corresponding to the combined increment $1 \& 2$ over the specimen's cross-section located mid-way between the $z^{-}$and the $z^{+}$face. (a) DVC reconstruction. (b) Model prediction, assuming anisotropic elastic behavior of the particles, weak particles-matrix interface and accounting for the micro-scale residual stress. (c) Absolute value of the difference between (a) and (b).

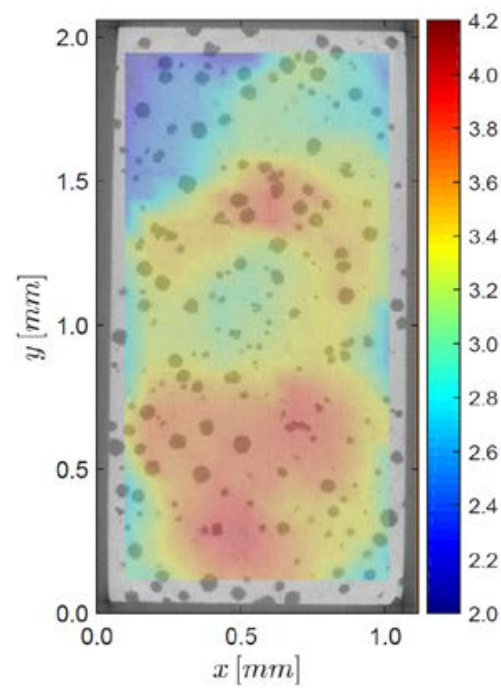

(a)

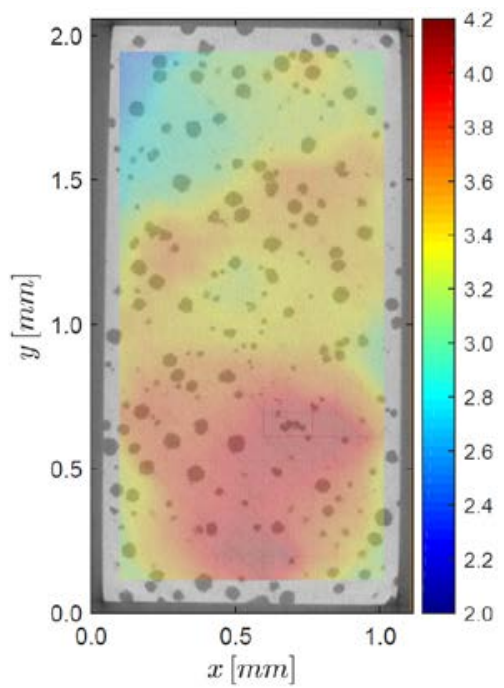

(b)

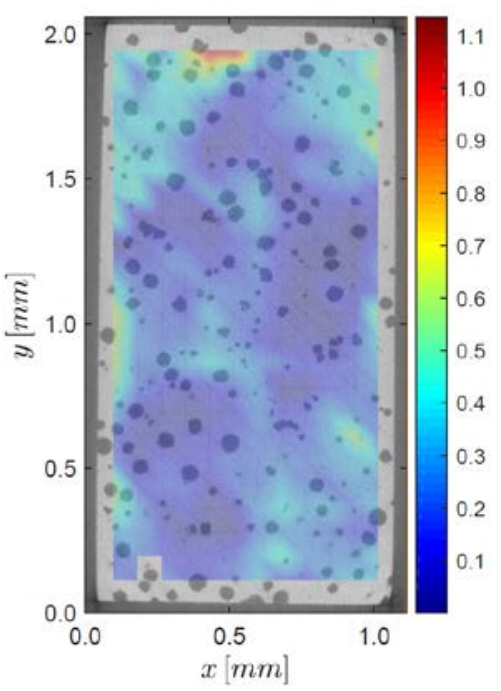

(c)

Figure 16 - Contour of the equivalent strain (in \%) corresponding to the increment 3 over the specimen's cross-section located mid-way between the $z^{-}$and the $z^{+}$face. (a) DVC reconstruction. (b) Model prediction, assuming anisotropic elastic behavior of the particles, weak particles-matrix interface and accounting for the micro-scale residual stress. (c) Absolute value of the difference between (a) and (b). 


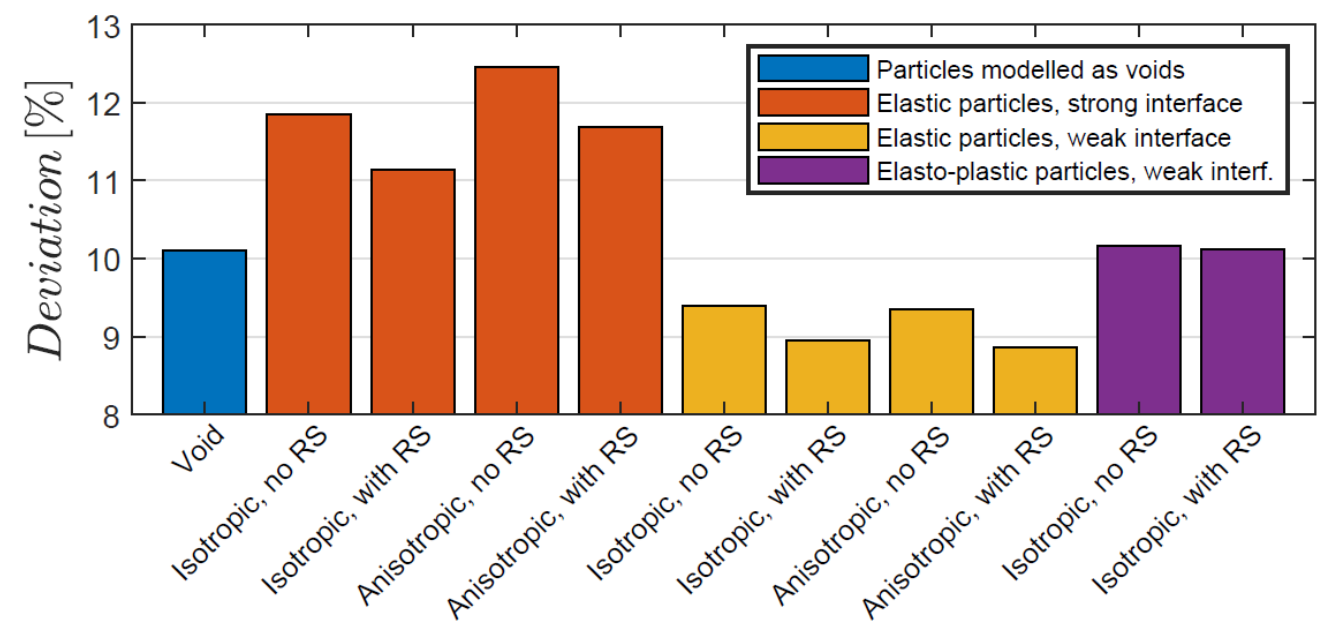

Figure 17 - Overall deviation - calculated by means of equation (12) - between the equivalent strain contour reconstructed via DVC and that predicted by the full model, over the specimen's cross-section located midway between the $z^{-}$and the $z^{+}$face. Data refer to the increment 3 .

\subsection{Full model vs. unit cell model}

As mentioned in the introduction, the third and final aim of this investigation is to clarify whether the significant effort required to develop the full model is justified. Concerning this, figure 18 shows the relative difference between the macroscale predictions of the much less expensive unit cell model and those of the full model, for the combined increment $1 \& 2$. It can be seen that the outcome of the two models is essentially equivalent in terms of lateral contraction, as the largest difference is only $4 \%$. This positive result might be a consequence of the fact that, in the unit cell, the macroscopic strain along the loading direction is prescribed as boundary condition. On the other hand, the tensile stress calculated with the unit cell model is systematically $7 \%$ - $13 \%$ larger than that calculated with the full model. One reason could be the spurious bending occurring during the tensile test as described in section 3.2.2, which is not accounted for in the unit cell model. To check this hypothesis, the full model simulations were repeated neglecting the contribution of bending to the displacement field prescribed as boundary condition. The gray bars in figure 18 indicate that, with this operation, the difference between the stress predictions of the two models decreases, but only by $\approx 1 / 3$, meaning that the main cause of such a difference is not the spurious bending. In this respect, figure 19 shows that the discrepancy between the two models seems to attenuate during the third increment, i.e. once plasticity 
has propagated extensively in the matrix. This suggests that the stress over-prediction of the unit cell model in the increment $1 \& 2$ is rather connected to the impossibility to account for the precise geometry of the initial plastic shear bands forming in the matrix, which is known to be strongly affected by the real distribution of the graphite particles in the microstructure (Kasvayee et al., 2017).

In order to evaluate the capacity of the unit cell model to isolate the effect of the individual micro-scale features considered in the present work, the predictions of both models for the eight simulations in which the particles are assumed elastic are analyzed within the framework of the design of experiments (Montgomery, 2012). Accordingly, the values of the predicted tensile stress - either at the end of increment $1 \& 2$ or at the end of increment 3 - are fitted with the following regression model:

$$
\begin{gathered}
\sigma=\sigma_{\text {avg }}+\beta_{A} x_{A}+\beta_{B} x_{B}+\beta_{C} x_{C}+\beta_{A B} x_{A} x_{B}+\beta_{A C} x_{A} x_{C}+ \\
+\beta_{B C} x_{B} x_{C}+\beta_{A B C} x_{A} x_{B} x_{C}
\end{gathered}
$$

where $\sigma_{\text {avg }}$ denotes the mean value, the $\beta s$ are the regression coefficients and the variables $x_{A}, x_{B}, x_{C}$ represent the factors under investigation, i.e. the initial stress state, the type of elastic behavior of the particles and the interface strength. These variables are defined on a normalized two-level scale $[-1,+1]$ and the simulation parameters associated with either the level -1 or +1 are defined in table 2 . The outcome of the fitting procedure is reported in table 3. Focusing on the combined increment $1 \& 2$, the very first observation that can be made is that the value of $\sigma_{\text {avg }}$ of the unit cell model is $17 \mathrm{MPa}$ larger than that of the full model, which confirms the systematic over prediction pointed out in the previous paragraph. Concerning the main effects of the three factors at hand, which are quantified by twice the values of $\beta_{A}, \beta_{B}$ and $\beta_{C}$, it can be stated that the two models lead to the same conclusion: The initial stress state and the interface type are the main factors controlling the tensile stress at the macroscopic yield point. However, the unit cell model indicates that the impact of the former is $50 \%$ higher than that of the latter, whereas, according to the full model, they have approximately the same impact. Remarkably, both models agree that during the hardening stage - see the results for the increment 3 in table 3 - the impact of the initial stress state becomes negligible while that of the interface type becomes even more pronounced. Conversely, the significant mismatch in the values of $\beta_{B}$ indicates that 
the two models differ in the prediction of the main effect of the particles' elastic behavior. In relation to this, it is worth noting that some factor interaction exists according to the full model, as revealed by the non-negligible value of the coefficient $\beta_{B C}$. This has to do with the particles' elastic behavior being significant when a strong interface is considered, but becoming negligible, despite $\beta_{B}=5.94 \mathrm{MPa}$, when the assumption of weak interface is made, due to the opposite values of $\beta_{B}$ and $\beta_{B C}$ which make the effect of the former factor vanish according to equation (13). Apparently, this type of interaction is not captured by the unit cell model, for which the effect of the particles' elastic behavior on the tensile stress is always negligible, no matter the value of the interface strength.

Table 2 - Definitions of factors and levels with respect to the regression model of equation (13).

\begin{tabular}{lcccccc}
\hline Factor & \multicolumn{2}{c}{ Initial stress state (A) } & \multicolumn{2}{c}{ Particles' elastic behavior (B) } & \multicolumn{2}{c}{ Interface strength (C) } \\
\hline Level & -1 & +1 & -1 & +1 & -1 & +1 \\
\hline Definition & No RS & With RS & Isotropic & Anisotropic & Strong & Weak \\
\hline
\end{tabular}

Table 3 - Coefficients of the regression model of equation (13). The full model data relate to simulations performed neglecting the influence of the spurious bending. Units are MPa.

\begin{tabular}{cccccccrr}
\hline Model type & $\sigma_{\text {avg }}$ & $\beta_{A}$ & $\beta_{B}$ & $\beta_{C}$ & $\beta_{A B}$ & $\beta_{A C}$ & $\beta_{B C}$ & $\beta_{A B C}$ \\
\hline \multicolumn{7}{c}{ Combined increment 1\&2 } \\
\hline Full model & 257.6 & -8.72 & 1.43 & -8.36 & -1.10 & 2.56 & -2.35 & 0.21 \\
Periodic unit cell & 274.5 & -13.12 & 0.20 & -7.07 & 0.07 & 1.91 & -0.29 & -0.08 \\
\hline \multicolumn{8}{c}{ Increment 3 } \\
\hline Full model & 414.9 & 1.12 & 5.94 & -38.53 & 0.11 & 1.01 & -6.11 & 0.04 \\
Periodic unit cell & 405.2 & 0.16 & -0.22 & -44.99 & -0.03 & 0.33 & 0.14 & 0.03 \\
\hline
\end{tabular}




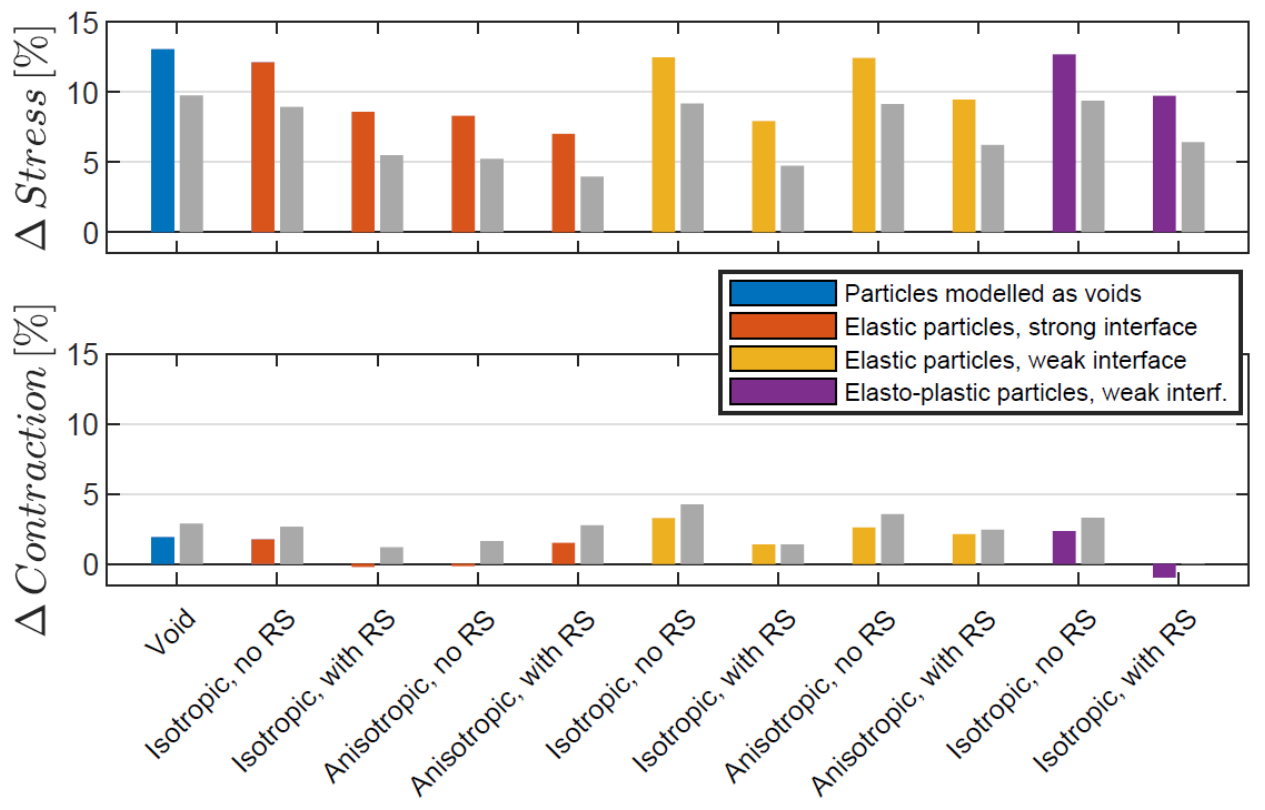

Figure 18 - Relative difference between the values of tensile stress and lateral contraction predicted by the periodic unit cell and those predicted by the full model, at the end of the combined increment $1 \& 2$. The gray bars show the same quantity when the contribution of bending to the deformation of the full model is neglected.

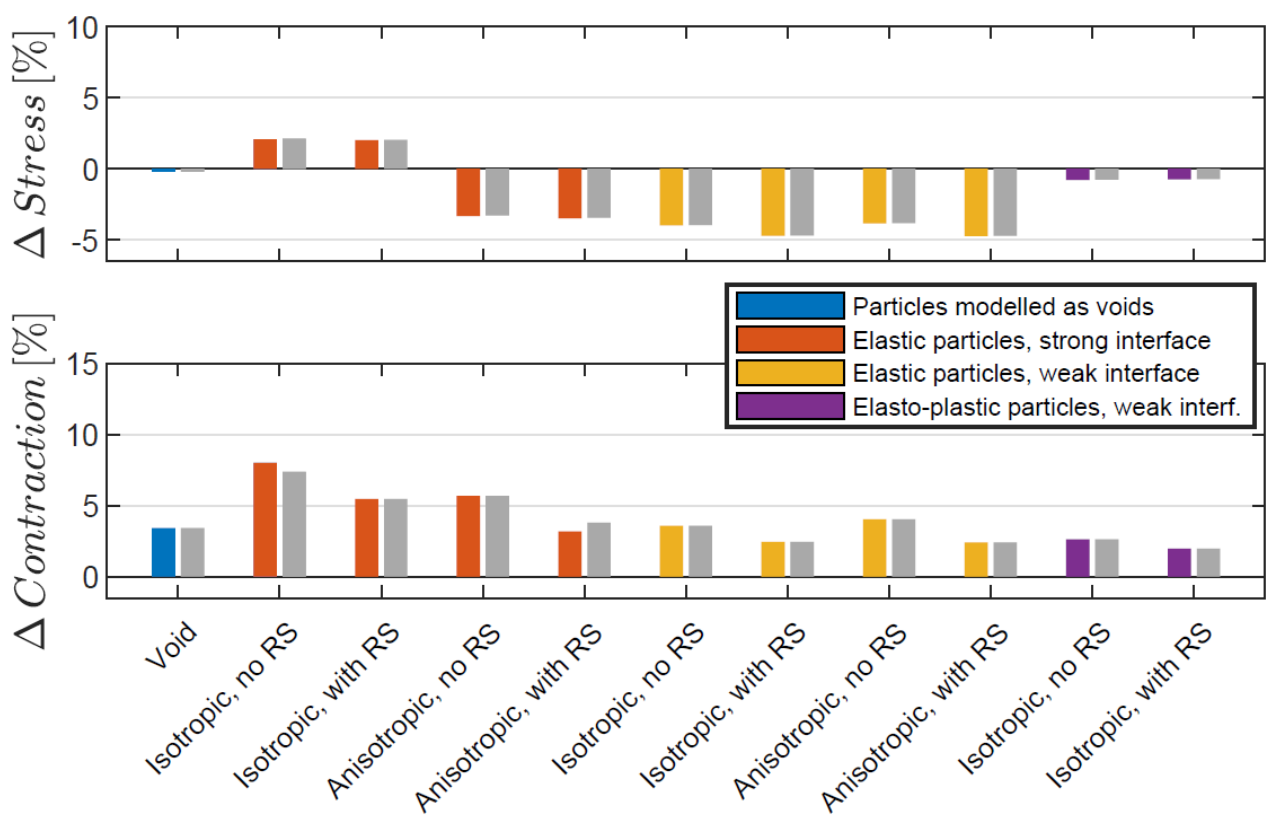

Figure 19 - Relative difference between the values of tensile stress and lateral contraction predicted by the periodic unit cell and those predicted by the full model, at the end of the increment 3 . The gray bars show the same quantity when the contribution of bending to the deformation of the full model is neglected. 


\section{Conclusions}

In the present work, a DCI specimen was imaged in-situ with X-rays during a tensile test and the microscale displacement field was reconstructed via digital volume correlation. A finite element model of the full microstructure between two cross-sections was setup and used to simulate both the formation of microscale residual stresses during manufacturing as well as the subsequent tensile deformation. Different options were investigated for the graphite particles' mechanical behavior and for the strength of the particles-matrix interface. By comparing the model predictions to the macroscale experimental data, to the microscale DVC-based strain field, and to the outcome of a simple periodic unit cell model, the following conclusions can be drawn:

1. At the macroscale, the error of the model is always minimized by assuming a weak interface between the particles and the matrix. If accounted for, the microscale residual stress field allows for a significant improvement in the model accuracy up to the macroscopic yield point and, in particular, allows justifying the early deviation of DCI from the ideal linear elastic behavior. Modelling the elastic anisotropy associated with the complex internal structure of the particles has an impact - positive - only at very small strains.

2. At the microscale, the strain field predicted by the model compares well with that reconstructed via DVC, and the effect of the microscale residual stress field, the particles' mechanical behavior and the interface strength is consistent with that recorded at the macroscale. However, this good agreement is only seen after significant plastic deformation of the specimen. Before, non-negligible differences between the predicted and the reconstructed strain fields exist, which can be explained mostly by the noise affecting the DVC data.

3. The greater effort required by the present experimental-computational approach appears justified, as, at the macroscopic yield point, a difference of $\approx 10 \%$ exists compared to the predictions of the unit cell model. The main reason seems to be the incapability of the latter to account for the real geometry of the microstructure and, 
to a lesser extent, for the actual deformation experienced by the specimen during the tensile test.

\section{Acknowledgements}

This work has been partially carried out within the frame of project "Optimised high performance ductile cast iron using 4D X-ray analysis and microstructural modelling', Danish Research Council for Independent Research, grant no. 10-093966. MAGMA GmbH and the Process Modeling Group at DTU are gratefully acknowledged for providing substantial financial support.

\section{Authors' contribution}

SF and YZ prepared the DCI specimen and performed the CT scans. TA and YZ carried out the DVC analyses. TA developed the finite element model and run the simulations with the help of JT. All authors discussed the results together. The final article was written by TA and revised and approved by all authors.

\section{References}

50th Census of World Casting Production, 2016. . Mod. Cast. - A Publ. Am. Foundry Soc. 25-29.

Aboudi, J., Arnold, S., Bednarcyk, B., 2012. Micromechanics of Composite Materials: a Generalized Multiscale Analysis Approach. Butterworth-Heinemann.

Andriollo, T., Fæster, S., Winther, G., 2018a. Probing the structure and mechanical properties of the graphite nodules in ductile cast irons via nano-indentation. Mech. Mater. 122, 85-95. https://doi.org/10.1016/j.mechmat.2018.03.010

Andriollo, T., Hattel, J., 2016. On the isotropic elastic constants of graphite nodules in ductile cast iron: Analytical and numerical micromechanical investigations. Mech. Mater. 96, 138-150. https://doi.org/10.1016/j.mechmat.2016.02.007

Andriollo, T., Hellström, K., Sonne, M.R., Thorborg, J., Tiedje, N., Hattel, J., 2018b. Uncovering the local inelastic interactions during manufacture of ductile cast iron: How the substructure of the graphite particles can induce residual stress concentrations in the matrix. J. Mech. Phys. Solids 111, 333-357. https://doi.org/10.1016/j.jmps.2017.11.005

Andriollo, T., Thorborg, J., Hattel, J., 2016a. Modeling the elastic behavior of ductile cast iron including anisotropy in the graphite nodules. Int. J. Solids Struct. 100-101, 523-535. https://doi.org/10.1016/j.ijsolstr.2016.09.023

Andriollo, T., Thorborg, J., Hattel, J., 2015. The influence of the graphite mechanical properties on the constitutive response of a ferritic ductile cast iron - A micromechanical fe analysis, in: Proceedings of the 8th International Conference on Computational Plasticity - Fundamentals and Applications, COMPLAS 2015.

Andriollo, T., Thorborg, J., Tiedje, N., Hattel, J., 2018c. Residual stresses around individual graphite nodules in ductile iron: Impact on the tensile mechanical properties. Mater. Sci. Forum 925, 465-472. 
https://doi.org/10.4028/www.scientific.net/MSF.925.465

Andriollo, T., Thorborg, J., Tiedje, N., Hattel, J., 2016b. A micro-mechanical analysis of thermo-elastic properties and local residual stresses in ductile iron based on a new anisotropic model for the graphite nodules. Model. Simul. Mater. Sci. Eng. 24, 055012(19pp). https://doi.org/10.1088/09650393/24/5/055012

Azeem, M.A., Bjerre, M.K., Atwood, R.C., Tiedje, N., Lee, P.D., 2018. Synchrotron quantification of graphite nodule evolution during the solidification of cast iron. Acta Mater. 155, 393-401. https://doi.org/10.1016/j.actamat.2018.06.007

Bargmann, S., Klusemann, B., Markmann, J., Schnabel, J.E., Schneider, K., Soyarslan, C., Wilmers, J., 2018. Generation of 3D representative volume elements for heterogeneous materials: A review. Prog. Mater. Sci. 96, 322-384. https://doi.org/https://doi.org/10.1016/j.pmatsci.2018.02.003

Bonora, N., Gentile, D., Pirondi, a., Newaz, G., 2005. Ductile damage evolution under triaxial state of stress: Theory and experiments. Int. J. Plast. 21, 981-1007. https://doi.org/10.1016/j.ijplas.2004.06.003

Bonora, N., Ruggiero, A., 2005. Micromechanical modeling of ductile cast iron incorporating damage. Part I: Ferritic ductile cast iron. Int. J. Solids Struct. 42, 1401-1424. https://doi.org/10.1016/j.ijsolstr.2004.07.025

Buljac, A., Trejo Navas, V.M., Shakoor, M., Bouterf, A., Neggers, J., Bernacki, M., Bouchard, P.O., Morgeneyer, T.F., Hild, F., 2018. On the calibration of elastoplastic parameters at the microscale via Xray microtomography and digital volume correlation for the simulation of ductile damage. Eur. J. Mech. A/Solids 72, 287-297. https://doi.org/10.1016/j.euromechsol.2018.04.010

Collini, L., Nicoletto, G., 2005. Determination of the relationship between microstructure and constitutive behaviour of nodular cast iron with a unit cell model. J. Strain Anal. Eng. Des. 40, 107-116. https://doi.org/10.1243/030932405X7692

Dassault Systèmes Simulia Corp., 2017. Abaqus 3DEXPERIENCE R2017x, Analysis User’s Guide section 37.1.10.

de Souza Neto, E., Peric, D., Owens, D.R.J., 2008. Computational methods for plasticity: theory and applications. Wiley.

Dong, M.J., Tie, B., Béranger, A.S., Prioul, C., François, D., 1997. Damage Effect on the Fracture Toughness of Nodular Cast Iron. Adv. Mater. Res. 4-5, 181-188. https://doi.org/10.4028/www.scientific.net/AMR.4-5.181

Eshelby, J.D., 1957. The determination of the elastic field of an ellipsoidal inclusion, and related problems. Proc. R. Soc. London A Math. Phys. Eng. Sci. 241, 376-396. https://doi.org/10.1098/rspa.1957.0133

Faris, F.E., Green, L., Smith, C.A., 1952. The thermal dependence of the elastic moduli of polycrystalline graphite. J. Appl. Phys. 23, 89-95.

Fernandino, D.O., Cisilino, A.P., Toro, S., Sanchez, P.J., 2017. Multi-scale analysis of the early damage mechanics of ferritized ductile iron. Int. J. Fract. https://doi.org/10.1007/s10704-017-0215-1

Feyel, F., Chaboche, J.-L., 2000. FE2 multiscale approach for modelling the elastoviscoplastic behaviour of long fibre SiC/Ti composite materials. Comput. Methods Appl. Mech. Eng. 183, 309-330. https://doi.org/https://doi.org/10.1016/S0045-7825(99)00224-8

Ghassemali, E., Hernando, J.C., Stefanescu, D.M., Dioszegi, A., Jarfors, A.E.W., Dluhoš, J., Petrenec, M., 2019. Revisiting the graphite nodule in ductile iron. Scr. Mater. 161, 66-69. https://doi.org/10.1016/j.scriptamat.2018.10.018

Grimvall, G., 1997. Cast Iron as a Composite: Conductivities and Elastic Properties. Adv. Mater. Res. 4-5, 31-46. https://doi.org/10.4028/www.scientific.net/AMR.4-5.31

Hild, F., Bouterf, A., Chamoin, L., Leclerc, H., Mathieu, F., Neggers, J., Pled, F., Tomičević, Z., Roux, S., 2016. Toward 4D mechanical correlation. Adv. Model. Simul. Eng. Sci. 3, 17. https://doi.org/10.1186/s40323-016-0070-z

Hill, R., 1963. Elastic properties of reiforced solids - Some theoretical principles. J. Mech. Phys. Solids 11, 357-372. https://doi.org/10.1016/0022-5096(63)90036-X

Hütter, G., Zybell, L., Kuna, M., 2015. Micromechanisms of fracture in nodular cast iron: From experimental findings towards modeling strategies - A review. Eng. Fract. Mech. 144, 118-141. https://doi.org/10.1016/j.engfracmech.2015.06.042

Iannitti, G., Ruggiero, A., Bonora, N., Masaggia, S., Veneri, F., 2017. Micromechanical modelling of constitutive behavior of austempered ductile iron (ADI) at high strain rate. Theor. Appl. Fract. Mech. 
https://doi.org/10.1016/j.tafmec.2017.05.007

Kasvayee, K.A., Ghassemali, E., Salomonsson, K., Sujakhu, S., Castagne, S., Jarfors, A.E.W., 2017. Strain localization and crack formation effects on stress-strain response of ductile iron. Mater. Sci. Eng. A 702, 265-271. https://doi.org/10.1016/j.msea.2017.07.018

Kohout, J., 2001. A simple relation for deviation of grey and nodular cast irons from Hooke's law. Mater. Sci. Eng. a A313, 16-23.

Kouznetsova, V., Brekelmans, W.A.M., Baaijens, F.P.T., 2001. An approach to micro-macro modeling of heterogeneous materials. Comput. Mech. 27, 37-48. https://doi.org/10.1007/s004660000212

Kuna, M., Sun, D.Z., 1996. Three-dimensional cell model analyses of void growth in ductile materials. Int. J. Fract. 81, 235-258. https://doi.org/10.1007/BF00039573

Kursa, M., Kowalczyk-Gajewska, K., Lewandowski, M.J., Petryk, H., 2018. Elastic-plastic properties of metal matrix composites: Validation of mean-field approaches. Eur. J. Mech. - A/Solids 68, 53-66. https://doi.org/https://doi.org/10.1016/j.euromechsol.2017.11.001

Lacaze, J., Castro, M., Lesoult, G., 1998. Solidification of spheroidal graphite cast irons-II. numerical simulation. Acta Mater. 46, 997-1010. https://doi.org/https://doi.org/10.1016/S1359-6454(97)00282-6

Lava, P., Cooreman, S., Coppieters, S., De Strycker, M., Debruyne, D., 2009. Assessment of measuring errors in DIC using deformation fields generated by plastic FEA. Opt. Lasers Eng. 47, 747-753. https://doi.org/10.1016/j.optlaseng.2009.03.007

Liu, J.H., Hao, X.Y., Li, G.L., Liu, G.S., 2002. Microvoid evaluation of ferrite ductile iron under strain. Mater. Lett. 56, 748-755.

Matouš, K., Geers, M.G.D., Kouznetsova, V.G., Gillman, A., 2017. A review of predictive nonlinear theories for multiscale modeling of heterogeneous materials. J. Comput. Phys. 330, 192-220. https://doi.org/10.1016/j.jcp.2016.10.070

Metzger, M., Seifert, T., 2015. Computational assessment of the microstructure-dependent plasticity of lamellar gray cast iron - Part I: Methods and microstructure-based models. Int. J. Solids Struct. 66, 184-193. https://doi.org/10.1016/j.ijsolstr.2015.04.016

Miao, B., Fang, K., Bian, W., Liu, G., 1990. On the microstructure of graphite spherulites in cast irons by TEM and HREM. Acta Metall. Mater. 38, 2167-2174. https://doi.org/10.1016/0956-7151(90)90084-T

Miao, B., North Wood, D.O., Bian, W., Fang, K., Fan, M.H., 1994. Structure and growth of platelets in graphite spherulites in cast iron. J. Mater. Sci. 29, 255-261. https://doi.org/10.1007/BF00356601

Monchoux, J.P., Verdu, C., Thollet, G., Fougères, R., Reynaud, A., 2001. Morphological changes of graphite spheroids during heat treatment of ductile cast irons. Acta Mater. 49, 4355-4362. https://doi.org/10.1016/S1359-6454(01)00230-0

Montgomery, D.C., 2012. Design and Analysis of Experiments, 8th ed. John Wiley \& Sons.

Morgeneyer, T.F., Helfen, L., Mubarak, H., Hild, F., 2013. 3D Digital Volume Correlation of Synchrotron Radiation Laminography Images of Ductile Crack Initiation: An Initial Feasibility Study. Exp. Mech. 53, 543-556. https://doi.org/10.1007/s11340-012-9660-y

Mostafavi, M., Bradley, R., Armstrong, D.E.J., Marrow, T.J., 2016. Quantifying yield behaviour in metals by X-ray nanotomography. Sci. Rep. 6, 1-7. https://doi.org/10.1038/srep34346

Muhmond, H.M., Fredriksson, H., 2014. Relationship Between the Trace Elements and Graphite Growth Morphologies in Cast Iron. Metall. Mater. Trans. A 45, 6187-6199. https://doi.org/10.1007/s11661014-2589-2

Mukherjee, K., Fæster, S., Hansen, N., Dahl, A.B., Gundlach, C., Frandsen, J.O., Sturlason, A., 2017. Graphite nodules in fatigue-tested cast iron characterized in 2D and 3D. Mater. Charact. 129, 169-178. https://doi.org/10.1016/j.matchar.2017.04.024

Ostoja-Starzewski, M., 2006. Material spatial randomness: From statistical to representative volume element. $\begin{array}{llll}\text { Probabilistic } \quad \text { Eng. } & \text { Mech. 112-132. }\end{array}$ https://doi.org/https://doi.org/10.1016/j.probengmech.2005.07.007

Pedersen, K.M., Tiedje, N.S., 2008. Graphite nodule count and size distribution in thin- walled ductile cast iron. Mater. Charact. 59, 1111-1121.

Peric, D., 1993. On a class of constitutive equations in viscoplasticity: formulation and computational issues. Int. J. Numer. Methods Eng. 36, 1365-1393.

Pina, J.C., Kouznetsova, V.G., Geers, M.G.D., 2015. Thermo-mechanical analyses of heterogeneous materials with a strongly anisotropic phase: the case of cast iron. Int. J. Solids Struct. 63, 153-166. 
https://doi.org/10.1016/j.ijsolstr.2015.02.048

Pina, J.C., Shafqat, S., Kouznetsova, V.G., Hoefnagels, J.P.M., Geers, M.G.D., 2016. Microstructural study of the mechanical response of compacted graphite iron: An experimental and numerical approach. Mater. Sci. Eng. A 658, 439-449. https://doi.org/10.1016/j.msea.2016.02.017

Qin, H., Sun, Y., Liu, J.Z., Liu, Y., 2016. Mechanical properties of wrinkled graphene generated by topological defects. Carbon N. Y. 108, 204-214. https://doi.org/10.1016/j.carbon.2016.07.014

Rabold, F., Kuna, M., 2005. Cell model simulation of void growth in nodular cast iron under cyclic loading. Comput. Mater. Sci. 32, 489-497. https://doi.org/10.1016/j.commatsci.2004.09.016

Rodriguez, F.J., Boccardo, A.D., Dardati, P.M., Celentano, D.J., Godoy, L.A., 2018. Thermal expansion of a Spheroidal Graphite Iron: A micromechanical approach. Finite Elem. Anal. Des. 141, 26-36. https://doi.org/10.1016/j.finel.2017.11.012

Sjögren, T., Svensson, I.L., 2005. Studying elastic deformation behaviour of cast irons by acoustic emission. Int. J. Cast Met. Res. 18, 249-256. https://doi.org/10.1179/136404605225023117

Sonne, M.R., Thorborg, J., Hattel, J.H., 2017. Modelling the effect of coating on the stresses and microstructure evolution in chill casting of wind turbine main shafts. Wind Energy. https://doi.org/10.1002/we.2114

Theuwissen, K., Lacaze, J., Laffont, L., 2016. Structure of graphite precipitates in cast iron. Carbon N. Y. 96, 1120-1128. https://doi.org/10.1016/j.carbon.2015.10.066

Theuwissen, K., Lacaze, J., Véron, M., Laffont, L., 2014. Nano-scale orientation mapping of graphite in cast irons. Mater. Charact. 95, 187-191. https://doi.org/10.1016/j.matchar.2014.06.021

Theuwissen, K., Lafont, M.-C., Laffont, L., Viguier, B., Lacaze, J., 2012. Microstructural Characterization of Graphite Spheroids in Ductile Iron. Trans. Indian Inst. Met. 65, 627-631. https://doi.org/10.1007/s12666-012-0162-5

Toro, S., Sánchez, P.J., Blanco, P.J., de Souza Neto, E.A., Huespe, A.E., Feijóo, R.A., 2016. Multiscale formulation for material failure accounting for cohesive cracks at the macro and micro scales. Int. J. Plast. 76, 75-110. https://doi.org/https://doi.org/10.1016/j.ijplas.2015.07.001

Tsang, D.K.L., Marsden, B.J., Fok, S.L., Hall, G., 2005. Graphite thermal expansion relationship for different temperature ranges. Carbon N. Y. 43, 2902-2906. https://doi.org/10.1016/j.carbon.2005.06.009

Zhang, K.S., Bai, J.B., François, D., 1999. Ductile fracture of materials with high void volume fraction. Int. J. Solids Struct. 36, 3407-3425. https://doi.org/10.1016/S0020-7683(98)00157-7

Zhang, Y., Pang, J., Shen, R., Qiu, Y., Li, S., Zhang, Z., 2018. Investigation on tensile deformation behavior of compacted graphite iron based on cohesive damage model. Mater. Sci. Eng. A 713, 260-268. https://doi.org/10.1016/j.msea.2017.12.077

Zhang, Y.B., Andriollo, T., Fæster, S., Barabash, R., Xu, R., Tiedje, N., Thorborg, J., Hattel, J., Juul Jensen, D., Hansen, N., 2019. Microstructure and residual elastic strain at graphite nodules in ductile cast iron analyzed by synchrotron X-ray microdiffraction. Acta Mater. InPress. https://doi.org/10.1016/j.actamat.2019.01.038

Zhang, Y.B., Andriollo, T., Fæster, S., Liu, W., Hattel, J., Barabash, R.I., 2016. Three-dimensional local residual stress and orientation gradients near graphite nodules in ductile cast iron. Acta Mater. 121, 173-180. https://doi.org/10.1016/j.actamat.2016.09.009 